Meta

Journal des traducteurs

Translators' Journal

\title{
La composante russe du Nadsat et ses métamorphoses en français, en russe et en bulgare
}

\section{Elena Meteva-Rousseva}

Volume 64, numéro 2, août 2019

URI : https://id.erudit.org/iderudit/1068199ar

DOI : https://doi.org/10.7202/1068199ar

Aller au sommaire du numéro

Éditeur(s)

Les Presses de l’Université de Montréal

ISSN

0026-0452 (imprimé)

1492-1421 (numérique)

Découvrir la revue

Citer cet article

Meteva-Rousseva, E. (2019). La composante russe du Nadsat et ses métamorphoses en français, en russe et en bulgare. Meta, 64(2), 359-392. https://doi.org/10.7202/1068199ar
Résumé de l'article

Le présent article traite des problèmes particuliers que pose la composante russe dans la traduction du Nadsat, l'argot qu'Anthony Burgess a inventé pour les jeunes voyous de son roman dystopique A Clockwork Orange (1962). Celui-ci se range parmi les meilleurs romans écrits en anglais. Le russe est la composante dominante du Nadsat. Exotique pour un anglophone, il pose de sérieux problèmes en traduction, surtout si celle-ci se fait vers le russe ou vers une autre langue slave, comme le bulgare. L'analyse partira des motifs qui ont poussé Burgess à doter ses personnages de ce langage crypté et à choisir le russe pour le forger. Seront détaillées la composition de cette couche lexicale et son incorporation à l'anglais pour étudier par la suite les solutions qu'ont trouvées les traducteurs vers le français, le russe et le bulgare pour reproduire l'effet de ce lexique russifié dans leurs traductions. Nos observations porteront sur la traduction française de Georges Belmont et Hortense Chabrier, parue en 1972 sous le titre L'Orange mécanique, sur celles, vers le russe, de Vladimir Bošnâk et d'Evgenij Sinel'ŝikov, toutes deux parues en 1991, ainsi que sur les deux versions de la traduction bulgare de Mariana Ekimova-Melniška, publiées respectivement en 1991 et en 2009.
Ce document est protégé par la loi sur le droit d'auteur. L’utilisation des services d’Érudit (y compris la reproduction) est assujettie à sa politique d'utilisation que vous pouvez consulter en ligne.

https://apropos.erudit.org/fr/usagers/politique-dutilisation/ 


\title{
La composante russe du Nadsat et ses métamorphoses en français, en russe et en bulgare
}

\author{
ELENA METEVA-ROUSSEVA \\ Université de Sofia "St. Kliment Okhridski", Sofia, Bulgarie \\ emeteva@gmail.com
}

\section{RÉSUMÉ}

Le présent article traite des problèmes particuliers que pose la composante russe dans la traduction du Nadsat, l'argot qu'Anthony Burgess a inventé pour les jeunes voyous de son roman dystopique A Clockwork Orange (1962). Celui-ci se range parmi les meilleurs romans écrits en anglais. Le russe est la composante dominante du Nadsat. Exotique pour un anglophone, il pose de sérieux problèmes en traduction, surtout si celle-ci se fait vers le russe ou vers une autre langue slave, comme le bulgare. L'analyse partira des motifs qui ont poussé Burgess à doter ses personnages de ce langage crypté et à choisir le russe pour le forger. Seront détaillées la composition de cette couche lexicale et son incorporation à l'anglais pour étudier par la suite les solutions qu'ont trouvées les traducteurs vers le français, le russe et le bulgare pour reproduire l'effet de ce lexique russifié dans leurs traductions. Nos observations porteront sur la traduction française de Georges Belmont et Hortense Chabrier, parue en 1972 sous le titre L'Orange mécanique, sur celles, vers le russe, de Vladimir Bošnâk et d'Evgenij Sinel'ŝikov, toutes deux parues en 1991, ainsi que sur les deux versions de la traduction bulgare de Mariana EkimovaMelniška, publiées respectivement en 1991 et en 2009.

\begin{abstract}
This paper explores the Russian-sounding component in Nadsat, the 'slang' invented by Anthony Burgess for the juvenile thugs of his dystopian novel A Clockwork Orange and the challenges it poses in translation. The novel appeared in 1962 and ranks among the best novels written in English. Russian is the essential building block of the Nadsat. Enigmatic for English speaking people, it raises serious issues in translation, especially if the latter is rendered in Russian or in another Slavic language, such as Bulgarian. The analysis will start from the motives that prompted Burgess to endow his characters with a cryptic language and to choose Russian for building it. The composition of this lexical layer and its incorporation into English will be examined, in order to analyze after that the solutions found by the translators into French, Russian and Bulgarian to replicate the effect of this Russified vocabulary in their translations. Our observations will be based on the translation of Georges Belmont and Hortense Chabrier, published in 1972 in France under the title L'Orange mécanique, the translations of Vladimir Bošnâk and Evgenij Sinel'ŝikov, both issued in Russia in 1991, and the two versions of Mariana Ekimova-Melniška's translation, edited respectively in 1991 and 2009 in Bulgaria.
\end{abstract}

\section{RESUMEN}

El artículo trata de los problemas específicos que plantea el componente ruso en la traducción del Nadsat - la jerga que Anthony Burgess inventó para los jóvenes granujas de su novela distópica A Clockwork Orange. La novela fue publicada en 1962 y hace parte de las 100 mejores novelas escritas en inglés. El ruso es el componente dominante del Nadsat. Enigmático para un anglohablante, plantea serios problemas de traducción sobre todo cuando es hacia el ruso o hacia otra lengua eslava como el búlgaro. El análisis 
partirá de los motivos que impulsaron a Burgess a dotar sus personajes de este críptico lenguaje y a recurrir al ruso para forjarlo. Será detallada la composición de esta capa léxica, su incorporación al inglés, con el fin de estudiar las soluciones ofrecidas por los traductores al francés, al ruso y al búlgaro para reproducir el efecto de este léxico rusificado en sus traducciones. Nuestras observaciones se basarán en la traducción de Georges Belmont y Hortense Chabrier, publicada en 1972 en Francia bajo el título L'Orange mécanique, la de Vladimir Bošnâk y de Evgenij Sinel'ŝikov, ambas publicadas en Rusia en 1991, así como las dos variantes de la traducción de Mariana Ekimova-Melniška, publicadas en Bulgaria respectivamente en 1991 y en 2009.

\section{MOTS CLÉS/KEYWORDS/PALABRAS CLAVE}

A Clockwork Orange, Nadsat, langue inventée, lexique russifié, traduction littéraire A Clockwork Orange, Nadsat, invented language, Russified vocabulary, literary translation A Clockwork Orange, Nadsat, lengua inventada, léxico rusificado, traducción literaria

\section{Introduction}

Le roman dystopique d'Anthony Burgess, A Clockwork Orange ${ }^{1}$ (1962), se range parmi les meilleurs romans écrits en anglais ${ }^{2}$. L'histoire se passe dans un avenir proche, les années 1970. Le Nadsat (du suffixe qui forme les chiffres de onze à dix-neuf en russe) est la langue du narrateur de l'histoire, Alex, âgé de quinze ans, et de ses trois drougs, adolescents comme lui, qui, galvanisés par la drogue, s'amusent la nuit à cambrioler, à violer et à terroriser les gens. Le nom laisse déjà sous-entendre que le russe est la composante principale de cet idiome inventé. Il y cohabite avec l'anglais familier, le cockney rimé, des mots forgés par Burgess lui-même, le langage enfantin et l'anglais de la King James Bible (xvII ${ }^{\mathrm{e}}$ siècle), sans compter les nombreuses allusions à un large éventail d'ouvrages littéraires.

Le Nadsat, comme toute autre langue construite, pose de sérieux problèmes en traduction. Pour pouvoir les résoudre, le traducteur doit non seulement faire preuve d'une créativité équivalente à celle de l'auteur, mais il doit aussi prendre en compte la fonction que l'auteur a assignée à cette langue dans son texte, les principes qui ont présidé à la formation de celle-ci et le rôle qui a été attribué à ses composantes. Quelle est la fonction du Nadsat? Comment est-il structuré? Pourquoi le russe est-il son élément constitutif de base? Telles sont les questions essentielles auxquelles nous allons tenter de répondre, avant d'analyser les stratégies qu'ont adoptées les traducteurs de l'œuvre vers le français, le russe et le bulgare pour recréer en langue cible le Nadsat, l'argot des jeunes vauriens "de l'ère cosmique». Nous chercherons aussi à déterminer dans quelle mesure les traducteurs ont réussi à reproduire l'effet que recherchait l'auteur par l'utilisation du Nadsat dans l'œuvre.

Ce langage, de prime abord incohérent, qualifié par l'auteur lui-même d'«aventure linguistique», est en fait le résultat du travail bien réfléchi d'un esprit talentueux. Passionné de musique classique, lui-même compositeur, épris également des langues et de la littérature, linguiste, enseignant, critique littéraire, librettiste, traducteur, Burgess est considéré comme un des rares génies littéraires de notre temps. À sa mort, le 22 novembre 1993, Times le décrit comme l'«un des écrivains les plus intelligents et les plus originaux de sa génération $»^{3}$. Déjà assez difficile à déchiffrer par le lecteur, le Nadsat est une épreuve de taille pour le traducteur, une vraie pierre de touche, surtout quand celui-ci doit traduire vers le russe ou vers une autre langue slave. Quels sont, par principe, les problèmes que pose la création lexicale en traduction et quelles pourraient être les possibilités de les résoudre? 


\section{La création lexicale dans la langue littéraire et sa traduction}

\subsection{Emprunts et néologismes}

La création lexicale, ou la néologie, consiste à incorporer des expressions ou des mots nouveaux dans la langue, par déformation, dérivation, emprunt, etc., ou encore à donner un sens nouveau à un mot ou une expression existante (Humbley 2006). Qu'il s'agisse d'un emprunt ou d'un néologisme inventé à partir des éléments qu'offre la langue elle-même, ces mots introduisent dans le texte des éléments étrangers (ou étranges), appartenant «à des systèmes de référence "autres" [...] qui mettent en place un monde auquel l'accès n'est pas totalement dégagé» (Delesse 2012: 9). Cette altérité crée des obstacles en traduction. Les emprunts et les néologismes, tout en étant de nature différente, «se rejoignent en ce sens qu'[ils] posent le problème de leur “étrangéité” et de leur degré d'intégration dans une langue donnée» (Delesse 2012: 9).

À la différence des emprunts qui se sont déjà naturellement insérés dans la langue, les éléments étrangers que l'auteur «importe» dans son texte sont là pour marquer leur étrangéité. Étrangéité qui devrait se faire sentir également dans la traduction. La stratégie adoptée par le traducteur, précise Delesse (2012: 10-11), dépendra du degré d'intégration de l'emprunt à la langue source et du statut qu'il aurait pu avoir dans la langue cible, de l'effet que l'auteur a voulu obtenir sur son public en y ayant recours et de celui que le mot étranger produirait chez le lecteur de la traduction. Si le statut de l'emprunt est le même, il serait gardé, sinon, un autre emprunt qui aurait le même effet sur les destinataires de la traduction pourrait le replacer.

Qu'il s'agisse d'un néologisme ou d'un emprunt, pour rendre son effet dans la traduction, le traducteur aura à répondre à plusieurs questions : quelle est sa raison d'être dans le texte?, quel est le contexte dans lequel il apparaît?, quelle est sa fonction dans le texte?, en est-il un détail supplémentaire ou un élément significatif?, le mot est-il vraiment nouveau, ou bien il a tout simplement acquis une nouvelle signification?, à quelle langue étrangère renvoie l'emprunt?, quel est le rapport que celle-ci entretient avec la langue de l'original et avec celle de la traduction?, le mot nouveau est-il facile à déchiffrer ou non?, comment est-il structuré?, etc. La réponse à toute cette série de questions serait déterminante pour le choix de la stratégie traductive.

Anthony Burgess fait fusionner les problèmes que posent ces deux types d'altérité, puisqu'il forge la majorité de ses mots nouveaux à partir d'éléments empruntés au russe. Tous les procédés qui sous-tendent la création de la néologie (composition, dérivation, amalgame, déformation, déplacement de sens, etc.) jouent sur un mélange entre l'anglais et le russe. Ce qui nous amène à nous arrêter plus en détail sur les problèmes que pose l'hétérolinguisme en traduction.

\subsection{Création hétérolingue et sa traduction}

Rainier Grutman distingue l'hétérolinguisme, qu'il définit comme «la présence dans un texte d'idiomes étrangers, sous quelque forme que ce soit, aussi bien que de variétés (sociales, régionales ou chronologiques) de la langue principale» (Grutman 1997: 37), du bi-, multi- ou polylinguisme où «les langues sont séparées, juxtaposées, enfermées dans leur espace respectif», comme elles le sont dans les sociétés bi- ou polylingues (Grutman 2012: 51). 
Les néologismes hybrides de Burgess, qui confondent l'anglais et le russe et cohabitent avec des variétés diastatiques, diaphasiques et diachroniques de l'anglais, rangent A Clockwork Orange du côté des textes hétérolingues.

Peut-on traduire ces textes hybrides? Les réponses à cette question sont plus que pessimistes. Pour Jacques Derrida, traduire l'hybridité est une tâche carrément impossible:

Et la traduction peut tout, sauf marquer [...] cette différence de système de langues inscrite dans une seule langue; à la limite elle peut tout faire passer [...] sauf le fait qu'il y a, dans un système linguistique, peut-être plusieurs langues. (Derrida 1982: 134)

Certains linguistes, à commencer par Nida et Taber (1969/1982: 4), considèrent que l'intraduisibilité apparaît là où la forme devient significative pour le message: "Anything that can be said in one language can be said in another, unless the form is an essential element of the message.» Le problème concerne surtout les ouvrages littéraires où la forme de ce qui est dit est tout aussi et, par moments, même plus importante que ce qui est dit. Comment l'aborder? Jacqueline Henry, qui traite de la traduction des jeux de mots où la question de la traduisibilité se pose dans toute son acuité, résume en concluant que tout dépend de «la base même que l'on se donne, à savoir celle de la correspondance linguistique ou de l'équivalence textuelle». Dans le premier cas, comme le dit Henry (2003: 261), «l'on rejoint le camp des tenants de [l']intraduisibilité», mais «si l'on choisit l'autre conception de la traduction, [...] celle de l'équivalence, ou de l'analogie» la possibilité de trouver une solution au problème est grande.

De toute façon il ne s'agira pas d'une traduction au sens standard du terme, mais de «translational mimesis », comme l'appelle Sternberg (1981 : 225). Celui-ci consiste à représenter, à imiter (et non à reproduire) dans la traduction l'hétérolinguisme du texte source.

L'alternance des codes est toujours censée assumer une fonction dans un texte littéraire. Il est pourtant des cas où elle fait partie intégrante du sens du texte, où sans elle celui-ci "perdrait de la valeur» (Stratford 2008: 461), comme c'est le cas du Nadsat. Le traducteur devrait alors redoubler ses efforts pour reproduire le plus fidèlement possible l'effet visé par l'auteur.

Dans le cas du Nadsat, le rôle attribué au langage hybride dans le texte est doublé d'un côté ludique, reflétant, en plus, le pur plaisir que l'auteur éprouve à explorer les sonorités étrangères, à jouer sur plusieurs langues, à expérimenter avec les possibilités que celles-ci lui offrent. Ce qui complique encore la tâche du traducteur.

Quel que soit le rôle de l'hétérolinguisme dans le texte, pour que le traducteur puisse l'estimer à sa juste valeur et essayer de le reproduire avec le moins de pertes possible, il devrait suivre la recommandation de Mezei (1988: 14-15): « [...] we must consider as well the factors which influence the production of meaning in the source text and in the target text; [...] the function of both source and target texts. » Cela implique de prendre en compte le texte, la culture qui l'a fait naître et le cadre culturel qui accueillera la traduction.

Les célèbres Exercices de style 4 de Raymond Queneau, qui racontent une même histoire banale de 99 façons différentes, constituent un bon exemple de texte où la forme et le jeu avec le langage deviennent primordiaux. Leur traduction en italien par Umberto $\mathrm{Eco}^{5}$ peut servir d'inspiration au traducteur qui doit faire face aux jongleries linguistiques de l'auteur et, par extension, à tous ces cas où la forme prend 
une place prépondérante dans le texte. Eco lui-même commente les stratégies qu'il a adoptées pour rendre, ou plutôt adapter, en italien les «intraduisibles» exercices de Queneau: «[...] il ne s'agissait pas de traduire, tout au moins au sens courant du terme, mais de comprendre les règles du jeu que Queneau s'était données, puis de jouer la même partie dans une autre langue» (Eco 1983; cité et traduit par Henry) ${ }^{6}$.

Pour finir, nous nous référerons à Stratford, qui résume pour les textes hétérolingues que

le traducteur devra faire preuve d'une grande ouverture à l'Autre s'il veut que sa traduction soit elle aussi «ouverte»: "open to differences, open to varieties of meanings, open to both the original cultural referential system and the one in the process of being created» (Mezei 1988: 20-21). Pour ce faire, le traducteur ne doit pas craindre de jouer avec sa langue, de laisser l'Autre y pénétrer jusqu'à en altérer la structure. Même si une traduction polyglotte n'aura jamais un effet tout à fait identique à celui de l'original, elle aura le mérite d'avoir reconnu sa nature hétérogène et d'avoir osé la reproduire [...]. (Stratford 2008: 466)

Le problème se corse pourtant quand la langue cible de la traduction est celle des éléments hétérogènes imbriqués dans le texte source. Comment procéder alors?

\subsection{Ramener l'élément étranger à sa langue d'origine}

À notre époque postcoloniale, globalisante, où populations et langues se mélangent de plus en plus, les traducteurs d'ouvrages littéraires écrits dans une langue métissée recourent souvent à des traductions littérales quand ils traduisent vers une des langues qui participent au métissage. Breton et Tinchant-Benrahho (2014) révèlent de quoi il s'agit, en se référant au fromlostiano, langue inventée par Federico López Socasau et Ignacio Ochoa Santamaría dans leur livre From Lost to the River ${ }^{7}$ (1995) (traduction littérale de l'expression espagnole de perdidos al río, dont provient le nom de la langue). La formation du fromlostiano imite'celle du spanglish et d'autres langues métissés et, mis à part son caractère ludique et humoristique, se présente également «comme une réflexion d'ordre traductologique»:

la langue s'y construit [...] en traduisant littéralement de l'espagnol à l'anglais (ou inversement), sans tenter de conserver la cohérence ni le sens de la version source. Au-delà du jeu littéraire et de la caricature qui pousse à l'extrême le principe de l'«étranger dans la langue» et de l'approche «littéraliste sourcière», il s'avère que ce livre construit aussi une réflexion sur la traduction (celle des expressions imagées, des références culturelles...) et l'acte de réécriture qu'elle sous-tend, moins caricaturale qu'il n'y paraît. (Breton et Tinchant-Benrahho 2014: 6-7)

Cette stratégie est préférée par les traducteurs des ex-colonies «européenisées » quand ils traduisent vers la langue de l'ancienne métropole, pour imposer leur langue vernaculaire (Chan 2002: 63-65). À part les traductions littérales du lexique, on pourrait faire intervenir également la grammaire et la syntaxe de la langue étrangère (Martín Ruano 2003: 201).

Aux traducteurs du spanglish vers le français, c'est-à-dire vers une troisième langue, Breton et Tinchant-Benrahho (2014: 6) suggèrent comme solution possible de re-créer le caractère mixte et ludique de ce langage, en «mêlant le français à chacune des deux langues, ou à l'une des deux selon les cas de figure, en fonction de la maîtrise linguistique supposée du public attendu ». 
Les problèmes du rapatriement de l'élément étranger, comme tous les autres problèmes concernant la traduction d'une langue hybride, se posent en fait quels que soient l'origine, le type et le rôle de l'hétéroglossie. Les différentes tentatives pour les résoudre ne sont encore que des tâtonnements. Comme le signale Martín Ruano (2003: 202), elles ne font que poser des questions. Mais, continue-t-il, dans le domaine de la traduction, de la transformation non seulement linguistique, mais aussi culturelle, parfois les questions ont plus de valeur que les réponses.

Les traducteurs du Nadsat vers le russe ou vers une autre langue slave se heurteront à ce problème dans toute son acuité.

\section{A Clockwork Orange et le Nadsat ${ }^{8}$}

\subsection{La naissance du roman}

Ce livre naît comme réaction à une théorie qui se répand de plus en plus en Angleterre, vers la fin des années 1950 et au début des années 1960, face à la criminalité juvénile grandissante. Plutôt que d'incarcérer les jeunes délinquants avec les adultes, il serait préférable de leur faire subir un traitement psychologique et médicamenteux radical qui puisse les guérir du mal. À ce sujet, Burgess ${ }^{9}$ déclare: «I feared the possibility that the state was all too ready to take over our brains and turn us into good little citizens without a power of choice.»

Burgess traite de ce problème à travers le sort de son personnage principal, Alex. Après une longue série de viols, de cambriolages, de tabassages, Alex se retrouve en prison où, pour se faire amnistier, il accepte qu'on expérimente sur lui un nouveau traitement, une sorte de «lavage de cerveau », qui le rend allergique à la violence. Une fois sorti de prison, inoffensif, il se voit attaqué par ses anciennes victimes. Une tentative de suicide ratée l'amène à l'hôpital avec une commotion cérébrale, dont le traitement efface les réflexes conditionnés acquis en prison. Il retourne dans la rue avec un nouveau gang, mais, ayant déjà grandi, il n'a plus de goût pour ce genre de vie et se met à rêver de fonder une famille ${ }^{10}$.

À part le grand succès de l'adaptation du livre pour le cinéma, faite en 1972 par Stanley Kubrick, à part les scènes de violence, très controversées à l'époque, c'est aussi le langage des jeunes voyous, leur Nadsat, qui a contribué au succès de ce livre, traduit et réédité à plusieurs reprises jusqu’à nos jours partout dans le monde.

\subsection{Pourquoi Burgess a-t-il inventé ce langage énigmatique?}

Pour échapper aux contraintes de temps et de localisation. Tout argot vieillit avec le temps. En créant ce langage, Burgess le rend intemporel. Grâce à lui, en plus, l'histoire aurait pu se situer n'importe où dans le monde.

Par son Nadsat, Burgess entend également soumettre son lecteur à un «lavage de cerveau», pareil à celui que son personnage subit. Ses lecteurs au moins, dit-il, en auront appris quelques mots russes. Il voulait que son texte soit un exercice de «programmation linguistique» où le sens des 218 mots exotiques (Vincent et Clarke 2017: 253) serait progressivement clarifié par le contexte. C’est pourquoi il refusait catégoriquement qu'on ajoute un glossaire au texte qui invaliderait sa "programmation» et annihilerait l'effet du «lavage de cerveau» (Burgess 1990: 38). Dans une lettre aux 
frères Heinemann, les premiers éditeurs de A Clockwork Orange, il expliquait qu'il suffirait au lecteur de lire une quinzaine de pages pour maîtriser et savourer le langage expressif qu'est le Nadsat. Cette affirmation s'est retrouvée, par la suite, sur la quatrième de couverture du livre (Vincent et Clarke 2017: 249, 257; Evans 1971: 406).

Il est vrai que peu à peu le lecteur s'habitue à ce vocabulaire étrange qui se répète, commence à le comprendre, se transformant, comme le signale Lesiew (2015: 66), de spectateur en un des droogs d'Alex, en un de ses brothers, l'appellation, par laquelle celui-ci s'adresse aux lecteurs, en les intégrant de la sorte à son monde.

Ce langage étrange crée en plus une sorte d'écran protecteur à travers lequel les scènes de violence brutale, de viol apparaissent comme irréelles. Il permet au lecteur de suivre l'histoire sans en être profondément bouleversé, en le protégeant en même temps de ses propres vils instincts (Burgess 1990: 38).

Le Nadsat peut également être vu comme «anti-langage», s'opposant aux valeurs de la société (Vincent et Clarke 2017 : 251) et également comme une sorte de violence exercée sur la langue (Malamatidou 2017: 293).

Il est évident que cet argot inventé assume plusieurs fonctions dans le roman, qu'il s'incorpore à son sens, ce qui revient à dire qu'il mérite toute l'attention du traducteur. Celui-ci devrait mobiliser toutes ses capacités analytiques et créatives pour construire sa propre version du Nadsat qui puisse assumer ce rôle complexe.

\subsubsection{Pourquoi le russe est-il à la base de cet argot inventé?}

En 1961, Burgess visite Leningrad avec sa femme. Il s'intéressait à la langue et à la culture russe depuis son adolescence (Kan 2012 ${ }^{11}$ ) et ce voyage lui a donné l'occasion de rafraîchir ses connaissances. Avant de partir, il avait déjà esquissé l'histoire de son livre, mais n’arrivait pas à trouver la langue pour la raconter. Son séjour là-bas l'aide à résoudre le problème:

[...] and soon it flashed upon me that I had found a solution to the stylistic problem of A Clockwork Orange. The vocabulary of my space-age hooligans could be a mixture of Russian and demotic English, seasoned with rhyming slang and the gipsy's bolo. [...] And there was fine irony in the notion of a teenage race untouched by politics using totalitarian brutality as an end in itself, equipped with a dialect which drew on the two chief political languages of the age. (Burgess 1990:37)

Quand Burgess écrit son roman, la guerre froide bat son plein. En août 1961, est construit le mur de Berlin. En 1962, lors de la crise des missiles de Cuba, le monde se retrouve au bord de la guerre nucléaire. La société occidentale est envahie par la peur de la Russie. Evans (1971 : 409) explique que pour le lecteur anglais ou américain les mots d'origine slave connotent la dictature communiste, la société où les valeurs morales n'existent pas, où les gens ont perdu tout espoir. La présence du russe dans cet argot est, pour Evans, une sorte d'avertissement de la part de Burgess: voilà ce que peut devenir notre société si elle se communise comme en Union soviétique. Et pourtant un an après la publication de l'article d'Evans, le 17 février 1972, Burgess publie lui-même un article dans The Listener, dans lequel il précise:

The language [...] is not mere decoration, nor a sinister indication of the subliminal power that a Communist super-state may already be exerting... I chose Russian words because they blend better into English than those of French or even German. (Burgess 1972: 198) 
Il est peu probable pourtant, comme le remarque Jackson (2011:66), que Burgess n'ait pas pensé aux associations que les gens allaient faire entre l'argot des jeunes voyous, basé sur le russe, et le système soviétique brutal à l'époque.

Le texte ne dit pas comment les garçons ont appris cet argot. À la question d'un des psychiatres dans le roman sur la provenance de l'«idiome tribal» incompréhensible d'Alex son collègue répond: "Odd bits of old rhyming slang [...]. A bit of gipsy ${ }^{12}$ talk too. But most of the roots are Slav. Propaganda. Subliminal penetration» (Burgess 1962/2000: 86). Réplique qui laisse supposer que les jeunes sont soumis au matraquage d'une force subversive qui cherche à les pervertir (Lesiew 2015: 65).

L'explication du médecin donne-t-elle vraiment une idée de ce que représente le Nadsat? Vincent et Clarke (2017: 251-252) qui, par l'analyse de corpus, ont calculé la fréquence de tous les items qui s'écartent de l'anglais standard et qui, pour eux, devraient être considérés comme appartenant au Nadsat, donnent la réponse à cette question..$^{13}$

\subsubsection{Composition du Nadsat}

Plutôt que de repérer des mots manouches, Vincent et Clarke (2017: 252-254) ont délimité sept catégories de mots:

1) Lexique russifié:

a. Il domine de loin et constitue le noyau du Nadsat.

2) Morphologie créative:

a. Changement de la forme et/ou de la catégorie grammaticale du mot;

b. Exemples:

- appetitish pour dire appetizing (appétissant);

- clowny comme adjectif de clown;

- gorgeosity, substantif dérivé de l'adjectif gorgeous (suffixation inhabituelle); etc.

3) Mots composés à partir de mots standard anglais:

a. Exemples:

- ultra-violent;

- lipmusic (faire pfft de mépris); etc.

4) Archaïsmes:

a. Exemples:

- ashake;

- canst;

- thou/thee/thy/thine;

- knowest;

- redding, etc.

5) «Rhyming slang»:

a. Exemples:

- pretty polly, qui renvoie à lolly (argent);

- luscious glory, de upper storey (cheveux);

- twenty-to-one, employé à la place de fun (amusement), etc.

6) Troncations:

a. Exemples:

- guff, de guffaw (s'esclaffer);

- pee et em pour father et mother ( $p a$ et $m a$ ); etc. 
7) Langage enfantin («babytalk»):

a. La formation de ces éléments se fait de deux façons. Pour les mots polysyllabiques (apologies), on ajoute $\underline{-i}$, -ie ou -y à chaque syllabe. Dans le cas des mots monosyllabiques, on répète la syllabe ou seulement sa terminaison après $\underline{-i}$ ou $-\mathrm{w}$ :

b. Exemples:

- eggiweg;

- purplewurple;

- baddiwad;

- appy-polly-loggies; etc.

Parmi les mots tronqués, Vincent et Clarke rangent sinny, derivé de cinema, mais employé également au sens de film. Celui-ci aurait aussi pu être formé à partir du français ciné (prononcé à l'anglaise). Pochon (2010: 76) y voit en plus une association possible avec sin (péché). Comme le lavage de cerveau qui doit rebuter Alex de la violence se fait par des films et comme ce sont les films qui sont l'instrument par lequel l'État exerce son pouvoir manipulatoire, cette interprétation de l'origine de sinny est plausible. Les traducteurs français sont apparemment de cet avis, traduisant sinny par cinique, ce qui relie par un jeu paronymique cinéma à cynique.

Par rapport aux mécanismes de formation du langage enfantin, Vincent et Clarke (2017: 250, 252) voient le même principe s'appliquer au mot russe мальчик [mal'čik] (garçon), ce qui donne malchickiwick. Cette explication est probable, bien que [mal'čik] est bisyllabique et ne devrait donc pas suivre le modèle de déformation que les enfants font subir aux monosyllabes anglais. Nous sommes plus encline à partager l'avis des locuteurs natifs de l'anglais que nous avons consultés, qui y ont vu une allusion à l'adjectif wicked (méchant, mauvais), le $i$ étant associé à la marque russe du pluriel. D’ailleurs les traducteurs français ont probablement fait une association semblable en traduisant par maltchikicaïd.

De toute façon, dans la mesure où, comme le signalent Vincent et Clarke (2017: 249), Burgess est demeuré vague et imprécis sur son Nadsat et s'est gardé de donner toute indication concernant l'analyse des mots qu'il a forgés, différentes interprétations sont possibles. Il se peut qu'il l'ait fait notamment pour laisser libre cours à l'imagination de ses lecteurs.

À la systématisation du lexique déviant du Nadsat, Vincent et Clarke (2017: 252253) ajoutent les écarts syntaxiques, citant en exemple to do the ultra-violent on. Ils soulignent l'utilisation substantivée de ultra-violent, ainsi que l'emploi du verbe do, assez général, à la place de perpetrate, inflict ou commit, plus précis et à priori plus appropriés au contexte.

\section{Le vocabulaire russifié et son intégration à l'anglais}

La manipulation d'une ou de plusieurs langues à des fins artistiques est présente dans l'ensemble de l'œuvre de Burgess.

Yet language, and its infinite permutations and possibilities, permeates every one of Burgess's 33 novels. From his first-published book Time for a Tiger [...] to his posthumous final novel, Byrne, written entirely in $18^{\text {th }}$ century poetic forms, his entire career in fiction was dedicated to preserving what he called the "opacity" of language in the service of art. (Clarke 2017: 32) 
L'opacification est donc recherchée par Burgess comme moyen d'expression littéraire. Dans le cas de A Clockwork Orange, il a choisi le russe pour caractériser ses personnages, créer la distance jugée nécessaire et pour forcer ses lecteurs à faire un effort de compréhension. Cet effort se voit quand même assisté par un large éventail de procédés linguistiques et stylistiques qui permettent au lecteur d'accéder au sens, notamment de ses emprunts au russe. Premièrement, Burgess a, par moments, recours à des gloses: rooker (a hand, that is) (Burgess 1962/2000:4); litso (face, that is) (Burgess 1962/2000: 4); Bog or God (Burgess 1962/2000: 5). Deuxièmement, il emploie en alternance le Nadsat et l'équivalent anglais: Our pockets were full of deng [...] But, as they say, money isn't everything (Burgess 1962/2000: 3). Enfin, il répète les emprunts à différents endroits, où peut apparaître le mot anglais correspondant, ou encore il fait tout simplement découvrir leur sens par le contexte.

Pour analyser les traductions, nous allons commencer par révéler les mécanismes qui sous-tendent le mélange des deux langues, «les règles du jeu» que Burgess «s'était données", pour reprendre la métaphore d'Eco (1983; cité et traduit par Henry), dans ce métissage, pour voir, par la suite, comment les traducteurs s'en sont servis pour forger leur Nadsat, ou, comme le dit Eco, pour essayer de «rejouer la même partie dans leur langue», c'est-à-dire d'adapter, d'imiter les mécanismes mis en ouvre par Burgess pour la création du Nadsat.

Les mots russes, façonnés par l'orthographe anglaise, sont souvent estropiés pour mieux correspondre aux intentions de l'auteur, à ses aspirations ludiques. Ils se voient assimilés par l'anglais, se soumettent aux règles de la grammaire de celui-ci. À part le contexte et le cotexte et les autres procédés que nous avons mentionnés, c'est aussi la grammaire qui permet au lecteur anglophone d'accéder à la signification de ces mots, ou, du moins, d'essayer de la deviner. La grammaire anglaise sert de liant au lexique hétéroclite pour faire du Nadsat une langue à part entière et en assurer le fonctionnement. Par moments, les marques grammaticales des deux langues se mélangent. Ce mélange qui se joint à l'hybridité lexicale fait penser à l'interlangue qui caractérise l'apprentissage d'une langue étrangère, la «phase transitoire» de celuici, marquée d'«interférences (code mixing) et [d']alternances codiques (code switching)» entre sa langue native et celle qu'on apprend (Montagut 2014: 5).

\subsection{La composition de la couche lexicale russe dans le roman}

Nous allons commencer par la distinction que font Blonskytė et Petronienè (2013: 62-63) entre emprunts russes et argotismes russifiés, tous deux présents dans le Nadsat. Conçu pour être l'argot des jeunes voyous de l'avenir, le lexique de cette langue inventée est censé remplir les trois fonctions essentielles qui caractérisent tout langage argotique: identitaire (marquer l'appartenance à un groupe), cryptique (exclure l'autre du groupe) et ludique (jouer sur et avec les mots, leur sonorité, leur sens, faire preuve de créativité, de fantaisie) (Antoine 2004: 11). L'argot attribue de nouvelles significations aux mots, les fusionne, modifie leur forme par troncation, resuffixation ou redoublement, invente des mots nouveaux, etc. Les saillies d'esprit, les rapprochements phonétiques et associatifs étonnants, les dérivations étranges, mélangées d'emprunts aux langues étrangères, sont souvent à l'origine de son lexique.

Les emprunts reprennent et transfèrent les mots tels quels d'une langue à l'autre. Ils se subdivisent en trois catégories: emprunts intégraux, où la forme et la signification 
du mot étranger sont importées, avec différents degrés d'assimilation phonétique; emprunts hybrides, dont la forme est empruntée seulement en partie (morphemic substitution) et s'associe à une forme native et; calques, qui empruntent le sens des mots étrangers (semantic loans) ou qui traduisent les morphèmes ou les éléments qui les composent (loan translation) (Haugen 1950: 213-215). Burgess a eu recours à des mots russes élémentaires, ceux qu'on apprend quand on s'initie à une langue étrangère. Il leur fait subir presque toutes les déformations ludiques qui caractérisent le lexique de l'argot, mais des emprunts intégraux sont également présents dans son Nadsat.

Quelle que soit la forme sous laquelle les mots russes ou russifiés y figurent, ils sont là pour étrangéiser et opacifier le texte. Le degré de cette étrangéisation et de cette opacification dépend, dans une large mesure, de la façon dont ils ont été adaptés à l'anglais.

Sofia Malamatidou (2017: 296) étudie la façon dont les emprunts au russe qui constituent le Nadsat de A Clockwork Orange ont été rendus en français, en allemand, en grec et en espagnol, et comment leur adaptation à la grammaire de la langue d'accueil et le genre qui leur est attribué différent de ceux des emprunts naturels au russe dans ces quatre langues. Elle s'appuie sur deux paramètres pour juger de l'adaptation morphologique d'un emprunt: 1) le genre qui y est attribué et 2) les suffixes flexionnels. Dans le cas du genre, elle identifie deux types de facteurs qui guident son attribution: sémantiques (similitude du sens entre langue source et langue d'accueil) ou formels (similitude structurale phonétique ou morphologique avec les mots de la langue d'accueil). Citons comme exemple de facteur sémantique le genre d'un (quasi) équivalent dans la langue d'accueil qui suggérerait le sens de l'emprunt et comme facteur formel, la terminaison d'un mot. À noter que le genre comme paramètre est inapplicable à l'anglais, dont les substantifs inanimés, en général, ne sont pas genrés. Quant aux suffixes flexionnels, ils permettent de mesurer le degré d'adaptation du mot au système grammatical de la langue d'accueil.

Nous avons cerné les différentes formes sous lesquelles les mots russes apparaissent dans le Nadsat. Nous les avons séparées en deux grands groupes: emprunts et déformations du sens ou de la forme.

\subsubsection{Emprunts}

La transcription des emprunts, assez libre, parfois fantaisiste, n'est pas aléatoire, comme en témoigne le commentaire de Burgess par rapport au mot russe грудь [groud']: "The English word, in which four consonants strangle one short vowel, is inept for that glorious smooth roundness. Groodies would be all right» (Burgess 1990 : 38). L'orthographe même du mot fait allusion aux seins féminins. Les légères déformations phonétiques ressemblent à celles que ferait un étranger qui ne maîtrise pas bien la langue et qui écrit les mots au son, n'arrivant pas à bien démarquer les syllabes. 
TABLEAU 1

Exemples d'emprunts intégraux

\begin{tabular}{|c|c|c|c|}
\hline $\begin{array}{c}\text { Type } \\
\text { d'emprunts }\end{array}$ & Nadsat & Mot russe d'origine & $\begin{array}{l}\text { Sens du mot russe d'origine } \\
\text { et du Nadsat correspondant }\end{array}$ \\
\hline \multirow{10}{*}{$\begin{array}{l}\text { Emprunts } \\
\text { transcrits }\end{array}$} & devotchka & девочка [devočka] & petite fillette, jeune fille \\
\hline & baboochka & бабушка [babuška] & vieille femme \\
\hline & gloopy & глупый, глуп [glupyj, glup] & bête, stupide \\
\hline & oomny & умный [umnyj] & intelligent \\
\hline & droog & друг [drug] & copain \\
\hline & rassoodock & рассудок [rassudok] & esprit, cerveau \\
\hline & malenky & маленкий [malenkij] & petit \\
\hline & pitt & пить [pit'] & boire \\
\hline & veshch & вещь [ves's] & chose \\
\hline & sladky & сладкий [sladkij] & sucré, etc. \\
\hline \multirow{10}{*}{$\begin{array}{l}\text { Emprunts } \\
\text { dont la forme } \\
\text { phonétique est } \\
\text { plus ou moins } \\
\text { déformée }\end{array}$} & itty & идти [idti] & aller (à pied) \\
\hline & dobby & добрый [dobryj] & bon \\
\hline & eemya & имя [imâ] & nom \\
\hline & yeckat & ехать [ehat'] & aller (en auto), rouler \\
\hline & ookadeet & уходить [uhodit'] & partir, s'en aller \\
\hline & lovet & ловить [lovit’] & attraper, arrêter \\
\hline & bugatty & богатый [bogatyj] & riche \\
\hline & bolshy & большой [bol'šoj] & grand \\
\hline & moodge & муж [muž] & mari, \\
\hline & messel & мысль [mysl'] & pensée, idée, etc. \\
\hline
\end{tabular}

\subsubsection{Déformations du sens ou de la forme des mots russes}

Les déformations ludiques que subissent les mots russes résultent, dans la plupart des cas, d'un jeu subtil sur l'association avec la sonorité ou les significations de mots anglais, ce qui aboutit à un mélange translinguistique de significations. C'est dans ce métissage des langues que Burgess donne toute la mesure de ses capacités linguistiques et de son inventivité langagière. En voici quelques exemples:

- ptitsa (dе птица [ptica]; oiseau) pour signifier femme ou nana, par association à l'anglais chick;

- кораt (dе копать [kopat']; bêcher, creuser) est utilisé au sens d'apprécier ou comprendre, sous l'influence du sens familier de to dig (piger);

- vareeting (dе варить [varit']; cuire, faire bouillir, faire la cuisine) pour signifier arriver ou se passer, par association au sens argotique du verbe to cook:

- [...] see what was vareeting (Burgess 1962/2000: 27);

- soomka (dе сумка [sumka]; sac) renvoie au sens argotique de bag (teigne);

- dook (de дух [duh]; fantôme, esprit) se substitue à son équivalent ghost en anglais dans l'expression not the dook of an idea (pas l'ombre d'une idée) (Burgess 1962/2000: 23), prenant un sens qu'il n'a pas en russe, etc.;

- horrorshow, mot-valise qui, par paronymie, renvoie à l'adverbe xopoшo [horošo] (bien) prend le sens de super, excellent, magnifique, fameux, foutu, sacré, etc., et fait 
allusion au plaisir pervers qu'Alex et ses copains éprouvent à semer l'horreur. On en compte 110 occurrences dans le livre, dans lesquelles le mot assume différentes fonctions:

- Adjectif: horrorshow takings (Burgess 1962/2000: 10);

- Adverbe: knowing each other's style a bit too horrorshow (Burgess 1962/2000: $41)$;

- Substantif (rare) : Real horrorshow (Burgess 1962/2000: 40).

Certains de ces jeux translinguistiques procèdent par allusion. La paronymie, un peu éloignée, relie le mot russe голова [golova] (tête) à gulliver, qui renvoie à Gulliver's Travels de Jonathan Swift, satire sociale et politique du début du XvIII ${ }^{\mathrm{e}}$ siècle qui a également trouvé sa place parmi les cent meilleurs romans écrits en anglais (McCrum 2015). Gulliver se substitue à head au sens propre et figuré: gulliver of the beer (Burgess 1962/2000: 19); [he] laughed his gulliver off (Burgess 1962/2000: 15). L'association paronymique est doublée donc d'un jeu sur la polysémie translinguistique, etc. À ces jeux translinguistiques s'ajoutent d'autres déformations, typiques de l'argot.

TABLEAU 2

Glissement de sens

\begin{tabular}{|l|l|l|l|}
\hline \multicolumn{1}{|c|}{ Nadsat } & \multicolumn{1}{|c|}{ Sens dans le Nadsat } & \multicolumn{1}{c|}{ Mot russe d'origine } & \multicolumn{1}{c|}{$\begin{array}{c}\text { Sens du mot } \\
\text { russe d'origine }\end{array}$} \\
\hline platties & vêtements & платье [plat'e] & robe \\
\hline pougly & effrayé & пугливый [puglivyj] & craintif \\
\hline to gooly & marcher, s'avancer & гулять [gulât'] & se promener \\
\hline privodeet & mener quelque part, emmener & приводить [рrivodit'] & amener \\
\hline chasha & tasse & чаша [с̌aša]; чашка [čaška] & bol, tasse \\
\hline lubbilub & faire l'amour & любить [lûbit'] & aimer \\
\hline
\end{tabular}

TABLEAU 3

Troncations

\begin{tabular}{|l|l|l|}
\hline \multicolumn{1}{|c|}{ Nadsat } & \multicolumn{1}{|c|}{ Mot russe d'origine } & \multicolumn{1}{|c|}{$\begin{array}{c}\text { Sens du mot russe d'origine et } \\
\text { du Nadsat correspondant }\end{array}$} \\
\hline to slooshy & слушать [slušat'] & écouter \\
\hline to osoosh & осушать [osušat'] & sécher \\
\hline to cheest & чистить [čistit'] & laver, nettoyer \\
\hline to creech & кричать [kričat'] & crier \\
\hline to pony & понимать [ponimat'] & comprendre \\
\hline to skvat & схватить [shvatit'] & saisir, empoigner, attraper \\
\hline to smot & смотреть [smotret'] & regarder \\
\hline to razdraz & раздразнить [razdraznit'] & agacer \\
\hline to razrez & разрезать [razrezat'] & couper \\
\hline chello veck, veck & человек [čelovek] & homme \\
\hline cheena & женщина [ženŝina] & femme \\
\hline
\end{tabular}


Les troncations assimilent, d'un côté, les mots polysyllabiques russes à l'anglais, dont les mots sont, pour la plupart, brefs, et, de l'autre, donnent au récit le rythme recherché par Burgess (Pochon 2010: 29). Windle (1995: 165) fait remarquer qu'en fait, Burgess se préoccupait beaucoup plus de l'effet sonore des mots russes à l'oreille de son lectorat anglais que de leur signification concrète.

TABLEAU 4

Exemples de morphologie créative

\begin{tabular}{|l|l|l|l|l|l|}
\hline \multicolumn{1}{|c|}{ Nadsat } & \multicolumn{1}{|c|}{ CG } & \multicolumn{1}{|c|}{ Sens dans le Nadsat } & Mot russe d'origine & \multicolumn{1}{c|}{ CG } & $\begin{array}{l}\text { Sens du mot russe } \\
\text { d'origine }\end{array}$ \\
\hline to tolchock & verbe & battre & толчок [tolčok] & subst. & $\begin{array}{l}\text { poussée, choc, } \\
\text { secousse }\end{array}$ \\
\hline to smeck & verbe & rire & смех [smeh] & subst. & rire \\
\hline skorry & adv. & vite & скорый [skoryj] & adj. & rapide \\
\hline to vred & verbe & faire du mal, agresser & вред [vred] & subst. & préjudice, dégât \\
\hline viddy & subst. & regard (to have a viddy) & видеть [videt'] & verbe & voir \\
\hline dratsing & subst. & bagarre & драться [drat'sâ] & verbe & se battre, se colleter \\
\hline crasting & subst. & vol avec violence & красть [krast'] & verbe & voler \\
\hline govoreet & subst. & parole, conversation & говорить [govorit'] & verbe & parler \\
\hline
\end{tabular}

CG: catégorie grammaticale

Viddy et govoreet sont employés également comme verbes et tolchock, comme substantif (pour dire coup). Le mot crasting figure presque uniquement comme substantif, mais on trouve aussi to crast (verbe). La modification de la catégorie grammaticale du mot russe d'origine n'exclut donc pas de voir celui-ci employé comme en russe.

Oddy knocky, dérivé de l'adjectif одинокий [odinokij] (seul, solitaire), apparaît le plus souvent à la place du pronom own, comme dans l'expression on one's own: slooshy the radio or read the gazetta, all on my oddy knocky (Burgess 1962/2000: 27); a small sort of cottage on its own (Burgess 1962/2000: 17). Cet emprunt remplace également self dans ourselves, ce qui le fait varier en nombre: you or me on our oddy knockies (Burgess 1962/2000: 31).

À partir de droog est formé droogie, qui fonctionne comme diminutif (little droogie, voir Burgess 1962/2000: 40), comme adjectif, sous la forme de droogy (showing their shining droogy zoobies) (Burgess 1962/2000: 38), et comme adverbe (smiling very wide and droogie) (Burgess 1962/2000: 13). Cet adverbe, dont la formation est tout à fait inhabituelle, ne pose quand même pas de problème de compréhension, étant lié à l'autre adverbe wide par la conjonction and; de plus, comme le mot droog a déjà apparu à plusieurs reprises dans le texte, le sens sera facile à deviner.

\subsection{Adaptation des mots russes à l'anglais}

Un mot étranger peut pénétrer dans la langue sous sa forme originale et alors son degré d'adaptation à la langue d'accueil est bas. Si, par contre, la désinence du mot change par l'addition, l'effacement ou le remplacement d'un suffixe flexionnel, le degré d'adaptation à la langue d'accueil est élevé (Malamatidou 2017: 301). Selon 
Malamatidou (2017: 302-303), l'analyse statistique à laquelle a été soumis le corpus russifié du Nadsat démontre que les mots d’origine russe qui y acquièrent des désinences anglaises sont deux fois plus nombreux que les emprunts russes qui ont pénétré dans l'anglais par voie naturelle (28,9\% contre $14 \%)$. Burgess a donc voulu que le langage d'Alex et de ses droogs soit relativement adapté au système linguistique dominant, mais seulement jusqu'à un certain degré. Cela leur permet de se faire comprendre, tout en conservant leur indépendance.

Les racines russes se voient attribuer des terminaisons typiques de l'anglais: $-\mathbf{y}$ (to viddy, to pony, poogly, gloopy, jeezny, etc.); -ck (chelloveck, malchick, etc.); -er (rooker, dérivé de рука [ruka]; main, bras). Le pluriel des substantifs et des adjectifs est marqué par un -s d'après les règles de l'anglais: droogs, rassoodocks, devotchkas, vecks, pletchoes, bugatties, etc. Il y a des cas où le $\underline{-s}$ anglais est ajouté au -i du pluriel russe: lewdies (dе люди [lûdi]); malchickies (мальчики [mal'čiki]); otchkies (очки [ос̌ki]; lunettes); zoobies (зубы [zuby]; dents); etc. La conjugaison des verbes suit aussi les règles de la grammaire anglaise: to peet, peeting, peeted; to sloshy, slooshying; to yeckat, yeckatted; ookadeet, ookadeeted; govoreet(s), govoreeted, govoreeting; to razrez, razrezzed; etc. Comme en anglais, les adjectifs ne s'accordent pas en genre et en nombre:

- the starry old baboochkas (Burgess 1962/2000: 11);

- malenky armies (Burgess 1962/2000: 13);

- little malenky creeches (Burgess 1962/2000: 19).

Ils forment leur comparatif et leur superlatif comme en anglais: bolshy, bolshier, bolshiest; gloopy, gloopier, gloopiest; oomny, oomnier, oomniest; etc. Enfin, les mots russes sont intégrés dans des expressions figées anglaises:

- He looked a malenky bit poogly (Burgess 1962/2000: 6), où a malenky bit veut dire a little bit (un petit peu);

- with the nochy still molodoy (pour dire the night is still young; avoir la nuit devant soi) (Burgess 1962/2000: 16);

- me having a sladky tooth (pour dire to have a sweet tooth; j'ai un faible pour les sucreries) (Burgess 1962/2000: 31); etc.

\section{Les traducteurs et leurs traductions}

Nous allons voir comment les traducteurs du livre en français, en russe et en bulgare se sont pris pour relever le défi que Burgess leur a lancé par cette dominante slave du Nadsat, dans quelle mesure ils ont su sauvegarder les deux fonctions primordiales dont Burgess l'a chargée: étrangéiser et opacifier le texte. Si, de ce point de vue, les traducteurs français sont relativement favorisés car le russe est aussi impénétrable pour les Français que pour les Anglais, tel n'est pas le cas des lecteurs bulgares et encore moins des lecteurs russes pour qui l'effet recherché par Burgess sera complètement perdu. Pour ces derniers, les traducteurs se verront obligés de chercher des mécanismes compensatoires pour sauvegarder, au moins en partie, cet effet. 


\subsection{Qui sont ces traducteurs?}

La traduction française est due à Georges Belmont et Hortense Chabrier. Elle paraît en 1972 sous le titre L'orange mécanique. Les deux traducteurs ont eu la chance d'être en contact direct avec Burgess, qui a hautement apprécié leur travail. Pour lui, ce sont de «superbes traducteurs» (Burgess 1990: 368). Les deux sont bilingues et ont, à plusieurs reprises, travaillé en équipe, y compris pour traduire d'autres ouvrages de Burgess. Pour la traduction d'Earthly Powers (1980), paru sous le titre La Puissance des Ténèbres et consacré Meilleur Livre étranger 1981, ils ont remporté le prix Charles Baudelaire.

Les deux premières traductions du livre en Russie paraissent en 1991. Celle de Vladimir Bošnâk, rééditée à plusieurs reprises depuis, et celle d'Evgenij Sinel'ŝikov, d'abord publiée dans la revue Junost - version abrégée, assez libre du livre. Au moment où Bošnâk a entrepris la traduction de A Clockwork Orange, il avait déjà treize ans d'expérience comme traducteur et avait traduit des auteurs comme William Saroyan, William Faulkner, Kurt Vonnegut, Aldous Huxley, William Styron, etc. Ses traductions lui vaudront plus tard à deux reprises $(2005,2007)$ le prix de la revue de littérature étrangère Inostrannaâ literatura (Adamov 2015). Interprète, traducteur, enseignant, Evgenij Sinel'ŝikov était également hautement apprécié pour ses traductions. Agatha Christie, Raymond Chandler, Sydney Sheldon, Frederick Forsyth figurent parmi les nombreux auteurs qu'il a traduits.

La traduction bulgare sort sous la plume de Mariana Ekimova-Melniška en 1991. Elle revoit le jour, remaniée à fond, en 2009. Traductrice, journaliste, universitaire, Mariana Melniška est comptée parmi les meilleures traductrices d'anglais. Elle a permis au public bulgare d'avoir accès à plusieurs ouvrages marquants pour ne citer que The French Lieutenant's Woman de John Fowles, trois des livres de la série Harry Potter de J. K. Rowling et The Evening News d'Arthur Hailey.

\subsection{Le Nadsat en traduction}

\subsubsection{Le Nadsat en français}

La traduction du Nadsat vers le français pose le moins de problèmes. Ses deux fonctions essentielles seront faciles à conserver, le russe pouvant servir d'élément opacifiant et assumer son rôle d'écran tout aussi bien pour les Français que pour les Anglais. La marque que le français a laissée sur l'anglais pendant les trois siècles de domination normande, l'empreinte que, par la suite, l'anglais a laissée sur le français créent un rapprochement lexical entre les deux langues qui facilite également la tâche des traducteurs.

\subsubsection{Composition lexicale}

Georges Belmont et Hortense Chabrier suivent de très près la stratégie que Burgess a appliquée aux mots russes. Partout où c'est possible, ils les reprennent de l'original tels quels. La structure et les composantes de la couche lexicale russe sont, pour la plupart, conservées, l'orthographe étant adaptée à la prononciation française, en laissant transparaître, par endroits, celle de l'anglais pour suggérer l'association entre les deux langues que Burgess a recherchée. 
TABLEAU 5

Exemples d'emprunts

\begin{tabular}{|c|c|c|c|c|}
\hline Type d'emprunts & $\begin{array}{l}\text { Nadsat } \\
\text { anglais }\end{array}$ & $\begin{array}{l}\text { Nadsat } \\
\text { français }\end{array}$ & Mot russe d'origine & $\begin{array}{c}\text { Sens du mot russe } \\
\text { d'origine et du Nadsat } \\
\text { correspondant }\end{array}$ \\
\hline \multirow{4}{*}{$\begin{array}{l}\text { Emprunts } \\
\text { transcrits }\end{array}$} & malchick & maltchick & мальчик [mal'čik] & garçon \\
\hline & chelloveck & tchelloveck & человек [čelovek] & homme \\
\hline & choodessny & tchoudessny & чудесный [čudesnyj] & merveilleux \\
\hline & shoom & choum & шум [šum] & bruit \\
\hline \multirow{9}{*}{$\begin{array}{l}\text { Emprunts dont la } \\
\text { forme } \\
\text { phonétique est } \\
\text { plus ou moins } \\
\text { déformée }\end{array}$} & itty & Itter & идти [idti] & aller \\
\hline & yeckat & yékater & ехать [ehat'] & aller (en auto) \\
\hline & ookadeet & oukaditer & уходить [uhodit'] & partir, s'en aller \\
\hline & moodge & moudj & муж [muž] & mari, jules \\
\hline & yahzick & yachzick & язык [jazyk] & langue \\
\hline & ooko & ouko & yxo [uho] & oreille \\
\hline & nochy & notché & Ночь [nоč’] & nuit \\
\hline & otchkies & otchquises & очки [očki] & lunettes \\
\hline & oozhassny & oudzassny & ужасный [užasnyj] & terrible, etc. \\
\hline
\end{tabular}

En d'autres cas, on trouve des déformations du sens ou de la forme des mots russes. La parenté entre le français et l'anglais a permis aux traducteurs de conserver presque tous les jeux de Burgess sur la polysémie translinguistique. Ptitsa (du mot russe signifiant oiseau) a gardé son sens de femme ou nana, pouvant être associé au sens figuré de poulette (jeune fille, jeune femme). Kopater (dérivé de копать [kopat']; bêcher, creuser) est aussi utilisé au sens de piger, renvoyant probablement à se creuser (expression familière pour dire réfléchir intensément). Le sens de variter (dérivé de варить [varit'] ; cuire, faire bouillir, faire la cuisine) pourrait être associé au sens figuré de mijoter: voir ce qui se varitait (Burgess 1962/1972: 27). Le jeu sur la polysémie de bag avec soomka (сумка [sumka]; sac) est également conservé: You filthy old soomka (Burgess 1962/2000 : 48) devient Vieille dégueulasse, vieux soumka à patates! (Burgess 1962/1972: 112). Le Nadsat soumka à patates fait allusion à l'expression sac à patates qui, dans le langage familier, peut désigner une personne obèse ou mal habillée.

Le mot clé du livre, horrorshow (pour dire super, etc.), trouve son analogue en français dans le télescopage de la transcription du mot russe цар [tzar] avec le mot français terrible: je lui ai mis une bogne tzarrible sur l'ooko (Burgess 1962/1972: 14); On y allait tzarrible (Burgess 1962/1972: 34). Gulliver (pour dire tête) est repris tel quel: Momo s'est presque pété le gulliver de rire (Burgess 1962/1972: 38).

Les deux traducteurs français ont également ajouté quelques trouvailles de leur cru. Leur créativité interlinguistique a donné naissance à plusieurs mots-valises. Ceux-ci fusionnent un mot russe avec un mot français qui en suggère le sens:

platties (vêtements), du russe платье [plat'e] (robe), se transforme en platrusques (platties combiné à frusques);

- to lovet (attraper, arrêter), du russe ловить [lovit'], devient lovretter;

- gloop (bête, stupide), du russe глуп [glup], devient gloupp (dont la double consonne renvoie à l'anglais) ou gloupide (gloup combiné à stupide);

- dratsing (bagarre), du russe драться [drat'sâ] (se battre, se bagarrer), est rendu par dratse ou dratsarre (transcription de драться combiné à bagarre);

glaz (œil), du russe глаз [glaz], est traduit par glaze, mais aussi parfois par glazard (transcription de глаз combiné à regard), qui prend alors le sens de regard; etc. 
Plus curieux et évocateur est l'adjectif moriturusse, employé dans le sens de mort et bien mort, qui associe le participe latin moriturus (celui qui va mourir, moribond), à l'adjectif russe. Les autres types de déformations sont également repris ou imités.

\section{TABLEAu 6}

Exemples de glissement de sens

\begin{tabular}{|l|l|l|l|l|}
\hline $\begin{array}{c}\text { Nadsat } \\
\text { anglais }\end{array}$ & $\begin{array}{c}\text { Nadsat } \\
\text { français }\end{array}$ & \multicolumn{1}{|c|}{ Sens du Nadsat } & \multicolumn{1}{|c|}{ Mot russe d'origine } & $\begin{array}{c}\text { Sens du mot } \\
\text { russe d'origine }\end{array}$ \\
\hline to gooly & goulatier & marcher, s'avancer & гулять [gulât'] & se promener \\
\hline privodeet & privoditer & mener, emmener & приводить [privodit'] & amener \\
\hline chasha & chascha & tasse & чаша [с̌aša] & bol \\
\hline skazat & skaziter & dire & скажите [skažite], сказать [skazat'] & dites, dire \\
\hline
\end{tabular}

Aux verbes tronqués s’ajoutent des désinences françaises.

TABLEAU 7

Exemples de troncations

\begin{tabular}{|l|l|l|l|}
\hline Nadsat anglais & Nadsat français & Mot russe d'origine & \multicolumn{1}{|c|}{$\begin{array}{c}\text { Sens du mot russe d'origine } \\
\text { et du Nadsat correspondant }\end{array}$} \\
\hline to slooshy & sloucher & слушать [slušat'] & écouter \\
\hline to sloochat & slouchater & случаться [slučat'sâ] & arriver, se passer \\
\hline to osoosh & ozoucher & осушать [osušat'] & sécher \\
\hline to cheest & tchister & чистить [čistit'] & laver, nettoyer \\
\hline to skvat & skvater & схватить [shvatit'] & saisir, empoigner, attraper \\
\hline
\end{tabular}

Il y a une légère déformation phonétique dans slouchater où - $\check{c}$ [tch] devient -ch et dans ozoucher où $-s$ devient $-z$. To razdraz (de раздразнить [razdraznit']; agacer, énerver) a été conservé sous la forme de l'adjectif razedraze (pour dire bouleversé, furieux); comme en anglais (veck et chello veck), veck, se substitue par endroits à tchelloveck; zhina (épouse, femme) paraît être une fusion entre cheena (qui correspond à la deuxième moitié de женщина [ženŝina]) et жена [žena] (épouse, femme).

\section{Tableau 8}

\section{Exemples de morphologie créative}

\begin{tabular}{|l|l|l|l|l|l|l|}
\hline $\begin{array}{c}\text { Nadsat } \\
\text { anglais }\end{array}$ & $\begin{array}{c}\text { Nadsat } \\
\text { français }\end{array}$ & CG & Sens du Nadsat & Mot russe d'origine & CG & $\begin{array}{l}\text { Sens du mot } \\
\text { russe d'origine }\end{array}$ \\
\hline sneety & snity & subst. & rêve & сниться [snit'sâ] & verbe & $\begin{array}{l}\text { rêver, voir en } \\
\text { rêve }\end{array}$ \\
\hline tolchock & toltchoker & verbe & battre & толчок [tolčok] & subst. & $\begin{array}{l}\text { poussée, choc, } \\
\text { secousse }\end{array}$ \\
\hline to vred & vreder & verbe & $\begin{array}{l}\text { faire du tort, du } \\
\text { mal, agresser }\end{array}$ & вред [vred] & subst. & $\begin{array}{l}\text { préjudice, tort, } \\
\text { dégât }\end{array}$ \\
\hline crasting & craste & subst. & $\begin{array}{l}\text { vol accompagné } \\
\text { de violence }\end{array}$ & красть [krast'] & verbe & voler, dérober \\
\hline govoreet & govoritt & subst. & $\begin{array}{l}\text { parole, discours, } \\
\text { conversation }\end{array}$ & говорить [govorit'] & verbe & parler \\
\hline smot(ting) & smott & subst. & regard & смотреть [smotret'] & verbe & regarder \\
\hline
\end{tabular}


En parallèle des substantifs craste, govoritt et smott, on trouve aussi les verbes craster, govoriter et smotter, respectivement. De même, on trouve le substantif toltchoke (coup, gifle) en plus du verbe toltchoker.

Droog devient droug, dont dérive l'adjectif drougui : avec un sourire genre drougui (Burgess 1962/1972: 122). Le mot choum (dе шум [šum]; bruit) est employé comme adverbe: Puis il est allé de sa bidonske - «Ho! ho! ho!» raide choum (Burgess 1962/1972: 19), où raide choum veut dire très bruyamment.

Les mots russes, comme en anglais, s'intègrent dans des expressions figées:

- le mot malenky, dérivé de маленкий [malenkij] (petit), dans un malenky peu, pour dire un petit peu, comme dans Il a eu l'air un malenky peu pouglé (Burgess 1962/1972: $14)$;

- le mot vred, dérivé dе вред [vred] (mal, tort), dans faire vred à qqn (Burgess 1962/1972: 34), pour dire faire $d u$ mal, $d u$ tort; etc.

Certaines des expressions anglaises sont calquées:

- la notché est encore molodoï (Burgess 1962/1972: 36), calque de the nochy still molodoy;

- j'ai une dent sladky (Burgess 1962/1972: 73), calque de me having a sladky tooth; etc.

\subsubsection{Le rôle de la grammaire}

Malamatidou (2017: 303-304) signale que le français, par principe, adapte de façon marquée les mots qu'il emprunte. Elle attribue ce phénomène à l'attitude des Français à l'égard des éléments étrangers dans leur langue. Dans le Nadsat français, $36 \%$ des mots russes sont adaptés à la grammaire française. Ce pourcentage est légèrement plus élevé que dans le cas des emprunts russes naturels en français; en effet, $31 \%$ des mots russes qu'emprunte le français sont adaptés à la grammaire cible. Dans le Nadsat original anglais, 28,9\% des mots russes ont été adaptés à la grammaire anglaise, contre $14 \%$ dans le cas des emprunts naturels du russe en l'anglais. Ainsi, pour les deux traducteurs, il était plus important que le pourcentage d'adaptation du Nadsat français se rapproche de celui du Nadsat original que de respecter le rapport de celuici au pourcentage d'adaptation des emprunts naturels en anglais. Ils se souciaient davantage de conserver le degré de violation des règles de la langue, et par extension de celles de la société, que Burgess voulait pour ses jeunes voyous.

Comme Malamatidou (2017: 296) le fait remarquer, le facteur sémantique le plus susceptible de suggérer le sens d'un emprunt est le genre qui lui est assigné, si celuici concorde avec le genre de l'équivalent cible le plus proche de l'emprunt. Les traducteurs français ont joué sur le genre de la plupart des mots russes empruntés. Lorsque les genres du mot russe et de l'équivalent français divergent, ils donnent le plus souvent le genre du mot français à l'emprunt russe.

- la collocolle, pour dire cloche, dе колокол [kolokol], masc. (cloche);

- la golosse, pour dire voix, de голос [golos], masc. (cloche);

- le roukeur, pour dire bras, de рука [ruka], fém. (bras, main);

- la rouke, pour dire main, de рука [ruka], fém. (bras, main);

- la noga, pour dire jambe, de нога [noga], fém. (jambe, pied);

- le noga, pour dire pied, de нога [noga], fém. (jambe, pied). 
Mis à part le glossaire qui est ajouté à la traduction française, la distinction que les traducteurs français font entre bras et main, entre jambe et pied, est un des rares cas où l'intention de Burgess n'a pas été respectée. Pour celui-ci, le fait que le russe ne fasse pas cette distinction est une des raisons qui l'a poussé à choisir cette langue: «This limitation would turn my horrible young narrator into a clockwork toy with inarticulated limbs» (Burgess 1990: 38).

L'analyse statistique à laquelle a été soumis le Nadsat français révèle que près de $90 \%$ des mots d'origine russe prennent le genre de leur équivalent français. Pour les emprunts russes naturels en français, ce pourcentage atteint presque $60 \%$. Cela révèle l'importance que les traducteurs ont accordée au facteur sémantique pour la compréhension du texte (Malamatidou 2017: 300-301).

En ce qui concerne les suffixes flexionnels, significatifs pour l'adaptation d'un mot étranger au système grammatical de la langue d'accueil, les traducteurs appliquent aux emprunts russes le modèle français de formation des mots: pougly devient pouglé; nochy se transforme en notché; groody est rendu par groudné; etc. Les verbes russes sont également francisés et se soumettent au système de conjugaison des verbes français: sitôt qu'il a eu slouché; je l'ai crastée; j'ai itté; slouchatant; j'ai critché; etc. (Burgess 1962/1972: 52; 108; 110; 112; 118). Quant aux adjectifs, ils prennent la désinence française pour la formation d'adverbes: bolchoïment, etc.

Le pluriel est également formé avec un -s comme en français: dévotchkas, gullivers, glazzies, slovos, groudés, etc. Il est intéressant de noter qu'en parallèle de drougs apparaît la forme drouguis: Mes drouguis haletaient à tout va (Burgess 1962/1972: 106). Cette deuxième forme cumule le $-s$ du pluriel français et le $-i$, une des marques du pluriel russe (le pluriel de друг pourtant еst друзья [druz'â] en russe).

Par des marques grammaticales, les traducteurs ont également voulu suggérer la présence de l'anglais comme élément constituant du Nadsat. Certaines terminaisons de l'anglais sont conservées: dobby, sladky, maltchick, veck, etc. La double consonne, tout à fait inhabituelle en français, apparaît souvent: smott, gloupp, govoritt, collocolle, etc. De plus, la plupart des adjectifs d'origine russe sont repris tels quels de l'anglais et, comme en anglais, restent invariables au féminin et au pluriel: De la bolchoï yarblokoss gros comme toi; la malenky statue d'argent (Burgess 1962/1972: 55 ; 112). Le mot drinker renvoie également à l'anglais. Bogic (2017: 225) affirme que les traducteurs l'ont utilisé parce que piter (dе пить [pit']; boire) aurait pu faire allusion à pitié ou à piteux et parce que drink est entré dans l'argot français au XIX ${ }^{\mathrm{e}}$ siècle.

\subsubsection{Le Nadsat en russe}

Si les traducteurs français n'ont eu aucun problème à étrangéiser et à opacifier le texte par l'entremise du russe, les traducteurs russes de l'œuvre se voient obligés de chercher d'autres moyens pour obtenir l'effet visé par Burgess. En effet, le russe, ramené «à la maison», cesse d'être énigmatique et ne peut plus servir d'écran.

Pour les critiques littéraires soviétiques des années 1960 et 1970, le roman de Burgess dénonçait les tares de la réalité capitaliste. Et pourtant, il n'a pas été traduit et publié en russe. Cela s'explique, selon Alexandr Livergant ${ }^{14}$ (cité dans Kan 2012), par les nombreuses scènes de violence dans le roman; l'idéologie éditoriale soviétique refusait catégoriquement de publier des livres de ce genre. De plus, la façon dont Burgess s'exprimait, dont il posait le problème, ne correspondait pas à ce que les maisons d'édition russes auraient aimé voir. Néanmoins, les intellectuels russes 
connaissaient le roman. Il y avait même des discussions concernant la langue dans laquelle le Nadsat aurait pu être traduit en russe. Ces discussions ont abouti à l'idée qu'il fallait avoir recours à une langue d'un état encore plus totalitaire que l'URSS. Le chinois a été évoqué... (Livergant, cité dans Kan 2012).

La publication du livre en Russie n'a eu lieu qu'en 1991, après la "perestroïka». Deux versions, très différentes l'une de l'autre, paraissent. Il y a, d'une part, la traduction d'Evgenij Sinel'ŝikov, variante assez libre et écourtée du roman original, publiée tout d'abord dans le journal Ûnost', qui s'éloigne de la traduction au sens classique du terme. D'autre part, il y a celle de Vladimir Bošnâk, parue sous forme de livre, qui suit de près le texte. Les deux ont adopté des stratégies traductives fort différentes pour rendre les éléments hétérolingues du texte original.

Comment conserver en russe l'effet que Burgess a recherché en forgeant son Nadsat? Le problème est de taille et sa solution reste, pour l'instant, ouverte. Burgess, interrogé en 1970 sur la traduction de ses œuvres, a répondu ainsi à ce problème: "The Russians, of course, would have no difficulty at all; they would merely have to replace my Slavonic loan-words with English ones » (Burgess 1970: 1025) ${ }^{15}$.

\subsubsection{La composition lexicale}

Evgenij Sinel'ŝikov a respecté la consigne de Burgess. Son texte est émaillé d'anglicismes transcrits en cyrillique. On trouve des emprunts à proprement parler:

мани [mani], de money;

- эмьюзмент [èm'ûzment], de amusement;

фэшн [fèšn], de fashion;

- поршн [poršn], de portion;

- иксайтмент [iksajtment], de excitement;

- крэнкшафт моушн джесче [krènkšaft moušn džesče], de crankshaft motion gesture; etc.

Comme dans le cas des emprunts russes de Burgess, Sinel'ŝikov donne à certains mots du Nadsat russe une prononciation qui cherche à imiter celle d'un apprenant russe de l'anglais (néophyte): бразер [brazer], фазер [fazer], мазер [mazer], de brother, father et mother, respectivement; ван фор ол [van for ol], de one for all; таэд [taèd], de tired; криче [kriče], de creature; etc.

Des déformations de la forme et du sens, bien que plutôt isolées, sont également présentes.

- Morphologie créative:

- визитинг [viziting], dérivé de to visit, prend le sens de visite.

- Fusion de l'anglais et du russe:

- бредница [brednica], dérivé de bread et combiné à хлебница [hlebnica], pour dire panier à pain;

- найсовая [najsovaâ], dérivé de nice et affixé d'une terminaison féminine russe, pour dire belle ou jolie;

- рингать [ringat'], dérivé de to ring et affixé d'une terminaison verbale russe, pour dire sonner;

- свимать [svimat'], dérivé de to swim et combiné à плавать [plavat'], pour dire nager;

- страйкнул [strajknul], dérivé de to strike et combiné à стукнул [stuknul], pour dire frapper, donner un coup; 
- ридальня [ridal'nâ], dérivé de to read et combiné à читальня [čital'nâ], pour dire salle de lecture.

Il est des cas où la racine russe est anglicisée: лауд стонинг [laud stoning], dérivé de стон [ston] (gémissement). Les trouvailles de Burgess sont parfois reprises: драцинг [dracing], transcription de dratsing, lui-même dérivé de драться [drat'sâ] (se battre) et signifiant bagarre, etc. Quoique ses anglicismes soient moins nombreux que les mots russifiés dans le Nadsat original (le glossaire que le traducteur a ajouté à son texte ne compte que 140 mots anglais), l'étrangéisation du texte est bien réussie.

L'opacification l'est, par contre, moins. Même des lecteurs qui connaissent bien le russe peuvent ne pas comprendre certains éléments du Nadsat original anglais (par ex.: cheena, sharries, yarbles). Or, une bonne partie des anglicismes introduits par Sinel'ŝikov avaient déjà pénétré dans la langue russe pendant les années 1980-1990; ainsi, au moment de la publication de la traduction, ils étaient largement employés par les jeunes (Windle 1995: 166-167). Les formes lexicales et grammaticales hybrides, la conservation des marques grammaticales de l'anglais, la transcription estropiée des mots qui les rend plus difficilement reconnaissables, toutes ces stratégies sont une bonne solution, mais elles ne sont pas assez nombreuses pour recréer l'effet d'écran que Burgess visait.

Quant à l'autre traducteur, Vladimir Bošnâk, il a carrément rejeté l'idée d'introduire des mots anglais dans le texte russe. Pour lui, il s'agissait d'« une ineptie conceptuelle, car le russe a servi à l'auteur pour exprimer l'idée que le mal vient de l'Est, de l'Union soviétique, de la Russie, qui était considérée comme l'“empire du mal”" (Bošnâk 2010 : s.p.; notre traduction ${ }^{16}$ ). Bošnâk a choisi d'assaisonner son texte de mots russes plus ou moins déformés, anglicisés par endroits.

Je chiffrais ironiquement par moments, je posais des rébus simples au lecteur, en cherchant à conférer aux propos une sonorité pareille à celle qu'ils auraient dans la bouche d'étrangers pour qui le russe est une langue complètement inconnue. Prononcés par eux, ces propos écorcheraient légèrement l'oreille. Je confondais exprès les racines avec les suffixes. (Bošnâk 2010: s.p.; notre traduction ${ }^{17}$ )

Il a voulu «cacher» dans une certaine mesure ces mots en les transcrivant assez librement en alphabet latin. À l'époque, précise-t-il, la transcription du russe en alphabet latin était assez inhabituelle. Il dit avoir peut-être été le premier à le faire dans un texte littéraire (Bošnâk, cité dans Kan 2012). Depuis l'avènement des ordinateurs, cependant, ce genre de transcription est monnaie courante. Sont repris certains emprunts russes que Burgess a utilisés, dont l'orthographe est souvent modifiée:

- peet devient pitt;

- malchick se transforme en malltshik;

- devotchka devient devotshka;

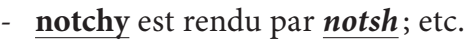

Certains mots déformés sont également repris: spatchka (sommeil), de спать [spat'] (dormir), est devenu spiatshka; dobby (bon), dérivé de добрый [dobryj], devient dobery. D'autres sont repris tels quels:

- glazzja, dе глаза [glaza] (уеux);

- ptitsa (au sens de femme, nana); 
- ruker, de рука [ruka] (main, bras);

morder, dérivé de морда [morda] (museau, gueule); etc.

Sont également imitées d'autres déformations auxquelles Burgess soumet les mots russes, par exemple la séparation de mots:

- Séparation de mots

- odi nochestvo, dérivé de одиночество [odinočestvo] (solitude);

- poni maiu, dérivé de понимаю [ponimaû] (comprendre, première personne du singulier);

- stari kashka, dérivé de старикашка [starikaška] (barbon), repris le plus souvent sous la forme kashka;

- Morphologie créative

- kritsh, dérivé de кричать [kričat'] (crier), employé à la place de cri, dont l'équivalent en russe est крик [krik];

- l'expression сделаю razterzats [sdelaû razterzats], au sein duquel razterzats provient de растерзать [razterzat'] (mettre en charpie, en pièces), prend le sens de massacrer; etc.

\subsubsection{Le rôle de la grammaire}

En ce qui concerne l'adaptation grammaticale, les langues slaves, par principe, ont une forte tendance à intégrer les mots qu'elles empruntent, à les adapter à leur système grammatical (Krumova-Cvetkova, Blagoeva et al. 2013: 190; Rozental', Golub et al. 1994: 86). Sans disposer de données statistiques concrètes, on peut tout de même affirmer que le Nadsat d'Evgenij Sinel'ŝikov contrevient à cette tendance. Les emprunts intégraux à l'anglais y sont beaucoup plus fréquents que dans la langue générale russe.

- дринкинг, и токинг, и тинкинг [drinking i toking i tinking], dérivé de drinking and talking and thinking;

- пэй визит [pèj vizit], dérivé de to pay a visit;

- дресст [dresst], dérivé de dressed;

- он лукс эт ас [on luks èt as], dérivé de looks at us;

- битс, битинг [bits, biting], dérivés de beats, beating;

- фор поршнз [for poršnz], dérivé de four portions;

- он риспондид [on rispondid], dérivé de responded; etc.

L'adaptation au système grammatical russe, relativement moins fréquente, est aussi présente.

- герла [gerla], dérivé de girl et affixé d'une terminaison féminine russe;

- фейсы [fejsi], dérivé de face et affixé d'une marque du pluriel russe;

- трахнуть по хэду [trahnut' po hèdu], dérivé de head et décliné au datif russe;

- в ее шопе [v ee šope], dérivé de shop et décliné au cas prépositif russe;

- со своими буками [so svoimi bukami], dérivé de book et décliné au cas instrumental russe; etc.

Par endroits, les marques des deux grammaires se cumulent. Dans le cas de бутсы [butsy], смайлзы [smajlzy], нейлзы [nejlzy] et айзы [ajzy], dérivés de boots,

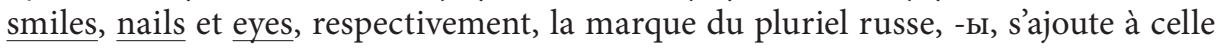
de l'anglais. Pour ce qui est de незанятыми хэндзами [nezanâtymi hèndzami], dérivé de hands, pluriel de hand, l'emprunt est décliné au cas instrumental russe; etc. 
Parfois, un mot anglais est introduit dans des expressions figées russes. Par exemple, dans l'expression плейс как плейс [plejs kak plejs], utilisée pour dire un endroit comme tant d'autres, le mot плейс [plejs] est dérivé de l'anglais place.

Dans les rares cas où le genre est pertinent, il constitue le facteur sémantique prédominant et le traducteur joue sur son attribution. L'emprunt à l'anglais prend le genre de son équivalent russe: dans в наше $n$ тайм [v naše tajm], le mot тайм [tajm], dérivé de time, est neutre, comme son équivalent russe, время [vremâ]; dans по всей $f$ стрит [ро vsej strit], стрит [strit], dérivé de street, est féminin, comme son équivalent russe, улица [ulica]; etc.

Rares sont les occurrences où c'est le facteur formel qui prend le dessus: dans этот гроссбук [ètot grossbuk], гроссбук [grossbuk], dérivé de gross book, est masculin. En effet, la plupart des mots qui se terminent par une consonne sont masculins en russe. L'équivalent russe de book, книга [kniga], est pour sa part féminin.

Quant à Vladimir Bošnâk, il a essayé d'évoquer l'anglais par la double consonne qui termine plusieurs verbes à l'infinitif, tout comme certains substantifs: $\underline{\text { nabliudatt }}$ (observer); vidett (voir); slyshatt (écouter); ponimatt (comprendre); tsepp (chaîne); vozhdd (leader); etc.

Des terminaisons anglaises sont aussi ajoutées aux mots russes, comme dans делали благовоспитанный $z$ drasting [delali blagovospitannyj zdrasting], qui veut dire nous saluions poliment (Burgess 1962/1991: 6). Le mot zdrasting est dérivé de здрасти [zdrasti] (bonjour, salut). Certaines des trouvailles de Burgess sont reprises de l'original: dratsing, krasting et toltshoking sont utilisés, surtout comme substantifs. Deux de ceux-ci, dratsing et krasting, sont réintégrés au russe par la voie du pluriel (dratsingi et krastingi, respectivement). Par endroits, c'est le pluriel anglais qui est utilisé: koreshes, de кореш [koreš] (pote); prestupniks, de преступник [prestupnik] (délinquant, malfaiteur).

Si ces mots se démarquent du reste du texte par leur transcription en alphabet latin, leur compréhension n'en souffre pas, car les mots latinisés respectent, dans la grande majorité des cas, les règles de la grammaire russe. Prenons pour exemple le syntagme с tseppju или britvoi, qui veut dire avec chaîne ou rasoir; les mots tseppju et britvoi sont dérivés respectivement de цепь [cep'] et de бритва [britva], déclinés au cas instrumental. De même, pour dire agiter les bras, on utilise le syntagme размахивал rukerami [razmahival rukerami], au sein duquel rukerami est décliné au cas instrumental. Le respect de la grammaire russe tient à quelques exceptions près: вокруг glazzjev, au lieu de la formulation correcte вокруг глаз [vokrug glaz] (autour des yeux). Par ailleurs, sapogoi et mozgoi, qui ont pris une désinence féminine, sont dérivés de substantifs masculins; сапогом [sapogom] et мозгом [mozgom] seraient donc les formes correctes au cas instrumental.

\subsubsection{Le nadsat en bulgare}

La traductrice bulgare Mariana Ekimova-Melniška s'est heurtée, bien qu'à un moindre degré, au même problème que celui auquel ont dû faire face les traducteurs russes. Le russe se rapproche assez du bulgare et, à ce titre, il ne pose pas trop de difficultés de compréhension pour les locuteurs bulgares. De plus, jusqu'à la chute du régime communiste en 1989 , deux ans avant la parution de la première édition de la traduction bulgare, les cours de russe étaient obligatoires à l'école. 
Mariana Melniška a quand même parsemé la première édition de sa traduction (1991) de mots russes, entremêlés seulement par endroits d'emprunts à l'anglais. Ces mots étrangers introduits dans le texte sont, dans la plupart des cas, adaptés au système grammatical bulgare et cette adaptation est la seule modification qu'ils subissent. Il n'y a pas de déformations supplémentaires et le texte est, dans sa totalité, assez compréhensible.

La nouvelle traduction, qui date de 2009, est, quant à elle, beaucoup plus chargée d'emprunts à l'anglais. Au dire de la traductrice (Ekimova-Melniška 2009: 7-8; 2010 : s.p.), elle s'est permis beaucoup plus de liberté dans cette deuxième version pour essayer de se rapprocher de la hardiesse et de l'érudition insondable de Burgess. Elle rappelle que le livre a été proscrit jusqu'en 1989 et que, au moment de la parution de sa première traduction, l'Union soviétique exerçait encore une certaine censure. Elle avoue que, à l'époque, la liberté de parole venant d'être acquise, son réviseur et elle n'avaient pas encore eu l'audace de trop jongler avec le russe. Elle ajoute que les maisons d'édition d'alors cherchaient à ménager au plus le lecteur, à ne pas le choquer. Ce n'est qu'avec l'affranchissement de la pensée que la deuxième édition du livre a été rendue possible.

La nouvelle version est, aux yeux de la traductrice, beaucoup plus osée, et met beaucoup plus le lecteur au défi. Celle-ci a été encouragée par le fait que, pendant les années qui ont suivi la parution de la première traduction, les Bulgares sont devenus plus réceptifs aux mots étrangers (Ekimova-Melniška 2009: 8; 2010: s. p.). Paradoxalement, la domination mondiale de l'anglais, qui fait en sorte que beaucoup de Bulgares l'apprennent et le parlent couramment, lui ôte le caractère énigmatique recherché par Burgess. Cela n’a cependant pas empêché certains lecteurs bulgares, habitués à ne pas faire trop d'effort en lisant, de se montrer très critiques de la nouvelle traduction $^{18}$.

\subsubsection{La composition lexicale des deux versions bulgares}

Les mots d'origine russe dans les deux versions bulgares, dont certains sont repris de l'original anglais, ne sont qu'adaptés à l'orthographe bulgare et, du fait de la parenté entre les deux langues, sont assez compréhensibles. On observe deux cas de figure par rapport aux mots russes introduits dans le Nadsat bulgare. D'une part, certains de ceux-ci se rapprochent beaucoup de leurs équivalents bulgares. Par exemple, расудок [rasudok], transcription du russe рассудок [rassudok], s'apparente au bulgare разсъдък $\left[\right.$ razs" $\left.^{\prime \prime} \mathrm{k}\right]$, tous deux voulant dire raison, esprit. La première section du tableau 9 montre d'autres exemples de ce type. D'autre part, la plupart sont tellement élémentaires que même des lecteurs bulgares qui n'ont jamais appris le russe peuvent les comprendre (voir deuxième section du tableau 9).

TABLEAu 9

Exemples de mots russes compréhensibles

\begin{tabular}{|l|l|l|l|}
\hline \multicolumn{1}{|c|}{ Nadsat bulgare } & \multicolumn{1}{|c|}{$\begin{array}{c}\text { Mot russe } \\
\text { d'origine }\end{array}$} & Équivalent bulgare & \multicolumn{1}{|c|}{$\begin{array}{c}\text { Sens des équivalents russe } \\
\text { et bulgare et du Nadsat }\end{array}$} \\
\hline Proximité des équivalents russes et bulgares \\
\hline молоко [moloko] & молоко [moloko] & млеко [mleko] & lait \\
\hline мозг [mozg] & мозг [mozg] & мозък $\left[\mathrm{moz}^{\prime \prime} \mathrm{k}\right]$ & cerveau \\
\hline
\end{tabular}




\begin{tabular}{|c|c|c|c|}
\hline кошка [koška] & кошка [koška] & котка [kotka] & chat \\
\hline $\begin{array}{l}\text { минуточка } \\
\text { [minutočka] }\end{array}$ & $\begin{array}{l}\text { минуточка } \\
\text { [minutočka] }\end{array}$ & минутка [minutka] & minute \\
\hline \multicolumn{4}{|c|}{ Mots russes élémentaires } \\
\hline сейчас [sejčas] & сейчас [sejčas] & сега [sega] & maintenant \\
\hline денги [dengi] & деньги [den’gi] & пари [pari] & argent \\
\hline очен [očen] & очень [očen'] & много [mnogo] & très \\
\hline друзя [druzâ] & друзья [druz'â] & приятели [priâteli] & copain \\
\hline рот [rot] & рот [rot] & уста [usta] & bouche \\
\hline вокруг [vokrug] & вокруг [vokrug] & около [okolo] & autour \\
\hline
\end{tabular}

Peu d'éléments du Nadsat bulgare sont dérivés de mots argotiques russes, comme c'est le cas pour каталажка [katalažka] (mot argotique, repris tel quel du russe, voulant dire prison) qui demanderait un effort de compréhension, même si le contexte en suggère le sens. La multiplication de tels termes argotiques ou familiers aurait pourtant pu aider à l'opacification du texte.

En plus des mots d'origine russe, la traductrice bulgare a multiplié, dans la deuxième version de son texte, les emprunts à l'anglais. Les mots empruntés à l'anglais sont pour la plupart très élémentaires, assez pour qu'une connaissance rudimentaire de cette langue soit suffisante pour les comprendre:

- дис тайм [dis tajm], dérivé de this time, pour dire cette fois;

- лейтър тунайт [lejt"r tunajt], de later tonight, pour dire plus tard ce soir;

- сори [sori], de sorry, pour dire désolé;

- вери уел [veri uel], de very well, pour dire très bien;

- дърти уърдс [d"rti u"rds], de dirty words, pour dire gros mots;

- съмтаймс [s"mtajms], de sometimes, pour dire parfois; etc.

La compréhension de certains autres emprunts demande une connaissance plus approfondie de l'anglais, mais ceux-ci sont peu nombreux:

- мърси [m"rsi], de mercy, pour dire pitié;

- сел [sel], de cell, pour dire cellule;

- снор [snor], de snore, pour dire ronfler;

- ръф $\left[\mathrm{r}^{\prime \prime} \mathrm{f}\right]$, de rough, pour dire brutal, brutalement;

- сует [suet], de sweat, pour dire sueur; etc.

Dans la deuxième version de la traduction bulgare, Melniška alterne dans son Nadsat les mots d'origine russe et d'origine anglaise. Souvent, une même réalité est décrite tantôt avec un emprunt au russe, tantôt avec un emprunt à l'anglais:

- молоко [moloko], transcrit du russe, mais aussi милк, de milk, pour dire lait;

- дърти [d"rti], de dirty, mais aussi грязни [grâzni], de грязный [grâznyj], pour dire sale;

- литъл [lit"l], de little, mais aussi маленкий [malenkij], pour dire petit;

- кричи [kriči], dérivé de кричать [kričat'], mais aussi скрийми [skrijmi], dérivé de to scream, pour dire il crie;

- голос [golos], du russe голос [golos], mais aussi войс [vojs], dérivé de voice, pour dire voix;

- инъф [in"f] dérivé de enough, mais aussi хватит [hvatit], dérivé de хватить [hvatit'], pour dire ça suffit. 
Il est des cas où le mot russe est là pour expliquer le sens du mot anglais. Par exemple, dans l'extrait suivant, l'emprunt au russe денги permet d'expliquer le sens de кеш, emprunt à l'anglais:

$\begin{array}{ll}\text { Bulgare: } & {[\ldots] \text { имаше съвсем малко кеш (деньги, значи) [...]. }} \\ \text { Transcription: } & {[\ldots . \text { imaše s"vsem malko keš (dengi, znači) [...]. }} \\ \text { Glose: } & {[\ldots] \text { il y avait très peu de cash (c'est-à-dire [den'gi]) [...]. }}\end{array}$

(Burgess 1962/2009: 23)

Mariana Melniška a imité Burgess en changeant, tout comme lui, la catégorie grammaticale, le sens ou la forme de certains emprunts qu'elle fait au russe ou à l'anglais (ceux qui étaient les plus fréquents dans le texte original):

- Morphologie créative:

- толчок [tolčok] (coup, poussée) reprend tel quel le substantif russe толчок. Comme Burgess l'a fait en anglais, un verbe est dérivé de celui-ci, qui apparaît sous les formes толчокна/xa [tolčokna/ha] (il a/ils ont frappé, poussé) et толчокван [tolčokvan] (frappé, poussé à plusieurs reprises);

- крастинг [krasting] et дратсинг [dratsing], transcription de crasting et dratsing, eux-mêmes dérivés des verbes russes красть [krast'] еt драться [drat'sâ], sont des substantifs (comme en anglais);

- Glissement de sens:

- платя [platâ], dérivé du russe платье [plat'e] (robe), est utilisé, tout comme par Burgess, au sens de vêtements;

- птица [ptica], qui veut dire oiseau en bulgare tout comme en russe, est utilisé au sens de femme;

- любилюбят ce [lûbilûbât se], оù пюбилюбят est dérivé du Nadsat anglais lubbilubbing (faire l'amour), qui lui-même provient du russe любить [lubit'] (aimer); etc.

Dans la deuxième version de sa traduction, Mariana Melniška a également eu recours à la morphologie créative:

- фистинг [fisting], dérivé du nom fist, pour dire donner des coups de poing; etc.

- той ме закюриоси [toj me zakûriosi] (Burgess 1962/2009: 61), utilisé pour dire il a éveillé ma curiosité, m’a intrigué, où закюриоси est dérivé de curious/curiosity;

- да ме изтолчокят аут [da me iztolčokât aut] (Burgess 1962/2009: 143), pour dire de me jeter dehors, оù изтолчокят аут est dérivé du russe толчок [tolčok] (poussée) et combiné à l'anglais out.

Les deux derniers exemples montrent des formes hybrides qui mélangent le bulgare soit avec le russe, soit avec l'anglais. Ceux-ci se trouvent dans les deux versions de la traduction bulgare. Prenons pour exemple l'extrait едни и тожи идеи, един и тожи колор [edni i toži idei, edin i toži kolor] (les mêmes idées, la même couleur) (Burgess 1962/2009: 58). S’y fusionnent les adjectifs indéfinis russes одни и те же [odni i te že] et один и тот же [odin i tot že] avec les adjectifs bulgares correspondants едни и същи [edni i s"šti] et един и същ [edin i s"šst']. De même, dans l'expression може да сте зафоргетили [može da ste zaforgetili] (Burgess 1962/1991: 5), utilisée pour dire vous pouvez avoir oublié, sont associés le verbe to forget et son équivalent bulgare забравям [zabravâm], dont le participe passé actif est забравил/и [zabravil/i]. Dans Burgess (1962/2009: 114), on trouve également l'expression без да може да мувне [bez da može da muvne] (sans pouvoir bouger), qui confond to move et le verbe bulgare correspondant да мръдне [da mr"dne], etc. Ces renvois aux équi- 
valents bulgares suggèrent le sens des verbes anglicisés. De tels jeux interlinguistiques auraient pu être multipliés.

Dans la deuxième version de la traduction bulgare, гъливер [g"liver], dérivé de gulliver, remplace par endroits голова [golova] (tête), emprunté au russe. Le mot clé de l'œuvre original, horrorshow, est transcrit par хорършоу [hor"ršou]. Par un jeu paronymique, celui-ci renvoie à l'adverbe russe xорошо [horošo] (bien); dans la bouche d'Alex, comme nous l'avons déjà mentionné, il prend le sens de super, foutu, sacré. On ne compte malheureusement que quelques occurrences de cette transcription, qui apparaît en alternance avec l'adverbe xopowo [horošo] (bien), repris du russe, avec l'adjectif diminutif хорошенкий [horošenkij] (du russe хороший [horošij]; bon, joli), et avec хорошичък [horošič"k], forme bulgarisée de хороший par l'ajout de la terminaison -чък [-č"k], qui forme le diminutif des adjectifs masculins bulgares. Ce faisant, la traductrice a édulcoré un trait qui caractérise le langage d'Alex et qui trahit le plaisir que celui-ci et ses drougs éprouvent à inspirer l'horreur.

Des expressions entières sont partiellement calquées, partiellement transcrites mélangeant bulgare, anglais et russe: нощта е още вери янг [noŝta e oŝe veri âng] (the night is still very young); a malenky bit (a little bit) est devenu маленкий бит [malenkij bit].

\subsubsection{Le rôle de la grammaire}

Par principe, le bulgare a tendance à adapter et à intégrer les mots étrangers, comme les autres langues slaves, ce qui caractéristique des langues conservatrices (Malamatidou 2017: 294). Malgré cette tendance linguistico-culturelle dominante, dans la version de 2009 de la traduction, le Nadsat bulgare comporte un assez grand nombre d'emprunts à l'anglais et au russe, qui sont transcrits dans leur forme grammaticale originale. Comme c'est le cas en russe, cette non-adaptation des emprunts, une violation des normes du bulgare, reflète la marginalisation sociale des jeunes délinquants de Burgess. Dans le roman original, au contraire, l'adaptation des emprunts au russe témoigne de cette marginalisation. Alors que $14 \%$ des emprunts naturels au russe sont adaptés au système grammatical de l'anglais, ce degré d'adaptation est deux fois plus élevé dans le Nadsat (Malamatidou 2017: 302). Les exemples suivants illustrent la non-adaptation des emprunts à la grammaire d'accueil au sein du Nadsat bulgare.

- Formation du pluriel:

- фистс [fists], transcription du pluriel fists, alterne avec фисти [fisti], transcription de fist combinée à la désinence du pluriel en bulgare $(-i)$, tous deux pour dire poing;

- $\underline{\text { xayces }}$ [hausez], transcription de houses;

- фейсис [fejsis], transcription de faces;

- тиърс [ti"rs], transcription de tears;

- $\underline{\mathbf{a и ̆}}$ [ajz], transcription de eyes;

- чилдрен [čildren], transcription de children; etc.

- Désinences verbales:

- прест [prest], de pressed;

- сърпрайзд [s"rprajzd], de surprised;

- мешают [mešaût], $3^{e}$ pers. pl. du russe мешать [mešat'] (empêcher, entraver);

- доломаем [dolomaem], $1^{\text {re }}$ pers. pl. du russe доломать [dolomat'] (casser, briser, fracasser); etc. 
- Terminaison de l'adjectif:

- болшое долгое $n$ канапе [bolchoe dolgoe kanape $n$ ], un grand canapé long; etc.

Rares sont les cas où la traductrice a volontairement glissé des fautes dans la marque grammaticale empruntée. Par exemple, dans l'expression извините мне [izvinite mne] (pardonnez-moi), меня [menâ], forme casuelle correcte de я [â] (moi), est remplacé par мне (cas grammatical incorrect). De même, elle utilise хочат [hočat] pour dire ils veulent, bien que хотят [hotât] est la forme correcte pour la $3^{\mathrm{e}}$ pers. du pluriel du verbe хотеть [hotet'] (vouloir).

La marginalisation que Burgess visait n'étant que partielle, il est normal que la plupart des emprunts dans la traduction s'inscrivent dans la tendance dominante d'adaptation des mots étrangers au système grammatical du bulgare. Les substantifs prennent les marques du pluriel et les articles définis du bulgare:

- глази [glazi], pour dire yeux, dérivé du russe глаз [glaz] (œil), dont la forme plurielle est глаза [glaza];

- покетите [poketite], pour dire poches, dérivé de pocket, affixé de -и [-i] (terminaison du pluriel bulgare) et de l'article défini pluriel -те [-te];

- найфове [najfove], pour dire couteaux, dérivé de knife, affixé de -ове [-ove] (terminaison du pluriel indéfini en bulgare);

- френдът [frend"t], pour dire l'ami, dérivé de friend, affixé de -ът [-"t] (article défini masculin singulier);

- голосьт [golos"t], pour dire la voix, dérivé de голос [golos] (cloche), affixé de -ът $[-" \mathrm{t}] ;$ etc.

Les adjectifs comparatifs suivent les règles du bulgare, par exemple по-биг [po-big] (plus big). Quant à по-лучше [po-lučše], il combine лучше [lučše], forme comparative de l'adverbe хорошо [horošo] (bien) et de l'adjectif хороший [horošij] (bon), et la marque du comparatif bulgare. Les terminaisons de certains adjectifs russes sont également adaptées au bulgare: болшаята [bolšaâta] (pour dire la grande), dérivé de болшая [bolšaâ], affixé de -та [-ta] (article défini féminin bulgare); etc.

Les verbes d'origine anglaise se voient attribuer toute la panoplie d'affixes, propres au bulgare, qui font varier l'aspect du verbe:

- зашаутих [zašautih], pour dire j'ai commencé à crier, dérivé de to shout, combiné au préfixe 3a- [za-], qui indique le début de l'action;

- скриймиха [skrijmiha], pour dire ils hurlaient, dérivé de to scream, adapté à l'aspect perfectif bulgare;

- зауорих ce [zauorih se], pour dire j'ai commencé à m’inquiéter, dérivé de to worry, combiné au préfixe 3a- [za-]; etc.

En ce qui concerne le genre des mots empruntés, c'est plutôt le facteur sémantique qui domine. Dans les exemples qui suivent, les emprunts anglais prennent le genre des mots bulgares correspondants; les adjectifs qui les qualifient, empruntés du russe, s'accordent alors en genre avec eux:

- джоук [džouk], féminin, est dérivé de joke, dont l'équivalent bulgare est шега [šega], féminin;

- En contexte: маленка джоук [malenka džouk];

- бук [buk], féminin, est dérivé de book, dont l'équivalent bulgare est книга [kniga], féminin;

- En contexte: маленка бук [malenka buk]; 
- кикинг [kiking], dérivé du substantif déverbal kicking, est utilisé au genre neutre, tout comme son équivalent bulgare, le substantif déverbal ритане [ritane] (coups de pied répétés);

- En contexte: болшое кикинг [bolchoe kiking].

L'influence du facteur formel se fait également sentir, bien que rarement. Citons l'exemple de фейс [fejs], genre masculin, dérivé de face. C'est la forme de cet emprunt qui a déterminé son genre, car en bulgare les mots masculins se terminent le plus souvent par une consonne (ici, -c [-s]). L'équivalent bulgare de face, лице [lice], est de genre neutre. De même, умбрела [umbrela], transcription de umbrella, est de genre féminin, les mots au féminin en bulgare se terminant par une voyelle (le plus souvent -a). Pour sa part, l'équivalent bulgare de umbrella, чадър [čad"r], est masculin.

Mariana Melniška a, en fait, beaucoup mieux réussi l'étrangéisation de la deuxième version de son Nadsat. Pour compenser la familiarité, plus ou moins importante, du russe pour le locuteur bulgare moyen, elle alterne emprunts au russe et emprunts à l'anglais, tout comme l'a suggéré Burgess, conservant l'idée de celui-ci de mélanger les deux langues dominantes de l'époque. L'opacification aurait pu être, par contre, un peu plus renforcée.

\section{Conclusion}

Dans le cas du Nadsat, ce n'est pas seulement l'hybridité de la langue qui pose problème, mais aussi, et surtout, le rôle que Burgess a assigné à la composante étrangère dans son texte. S'y ajoute également l'élément ludique, le plaisir de jongler avec les langues, de mélanger leurs sonorités et leurs significations. La langue exotique qu'est le russe aux yeux de l'anglophone devait servir d'écran, d'obstacle à franchir pour accéder au sens du texte. Elle demandait un effort de la part du lecteur; elle était censée le soumettre à une sorte de lavage de cerveau.

Georges Belmont et Hortense Chabrier, bien plus favorisés que les autres traducteurs, ont suivi de très près les «règles du jeu» que Burgess s'était données et ont pu reproduire, même calquer la plupart de ses inventions. Malamatidou (2017: 304) leur a pourtant reproché d'avoir donné la priorité au sens des mots Nadsat, d'avoir trop subi l'influence du texte source. En tâchant de ne pas s'en écarter, ils ont limité leur créativité, ce qui ne leur a pas permis de reproduire entièrement l'effet recherché pour le Nadsat original.

La difficulté a été la plus grande pour les traducteurs russes. En effet, de retour dans son pays d'origine, le russe ne peut plus remplir les deux fonctions principales qu'il est appelé à jouer dans le texte anglais. Burgess, en conseillant aux traducteurs russes de recourir à des emprunts à l'anglais, n’avait pas prévu l'internationalisation subséquente de l'anglais, qui l'empêcherait de servir d'écran. La stratégie de Bošnâk - s'en tenir au russe, en s'appuyant sur la transcription en alphabet latin et en ne déformant que quelques mots - est aussi loin de pouvoir reproduire cet effet. Evgenij Sinel'ŝikov, lui, a montré que la transcription des mots anglais peut servir pour étrangéiser le texte, mais qu'elle n'est pas suffisante pour l'opacifier.

La même remarque s'applique aux traductions de Mariana Ekimova-Melniška. Elle a bien su conserver l'étrangéisation du texte, surtout dans la deuxième version de sa traduction, mais l'opacification de celui-ci laisse encore à désirer. 
L'opacification demanderait aux traducteurs vers le russe, ou vers une autre langue slave, de mobiliser leur mémoire associative, leur imagination dans le maniement des deux langues. Il leur faudrait puiser dans les couches un peu plus profondes de la ou des langues étrangères, reprendre et multiplier les glissements de sens, les troncations, les distorsions, les formes hybrides, les jeux translinguistiques sur l'homonymie, la paronymie ou la polysémie entre les langues impliquées, créer des dérivations inhabituelles, etc., tout comme Burgess l'a fait pour ses emprunts russes. Comme le dit Chan (2002: 61): «The key is cross-fertilization, the release of verbal energy through the collision of words from different tongues.» Traduire littéralement des expressions figurées de l'anglais (et du russe) peut également être approprié.

En résumé, la traduction du Nadsat est un vrai défi lancé aux traducteurs. Pour le relever, ils doivent non seulement faire preuve d'une grande maîtrise de la langue, mais aussi donner libre cours à leur créativité, s'adonner au plaisir de jouer avec les langues et avec les associations qu'elles engendrent. Pour ce faire, ils doivent posséder une certaine liberté d'esprit, un tempérament badin et la riche culture d'Anthony Burgess.

\section{NOTES}

1. Les références aux éditions de l'original et des traductions sur lesquelles nous avons travaillé, ainsi qu'à des interviews ou des écrits où l'auteur ou les traducteurs révèlent leurs points de vue sur le roman se trouvent en annexe.

2. McCrum, Robert (17 août 2015): The 100 best novels written in English: the full list. The Guardian. Consulté le 20 décembre 2018, <https://www.theguardian.com/books/2015/aug/17/the-100-bestnovels-written-in-english-the-full-list>.

3. Notre traduction: "one of the cleverest and most original writers of his generation». ANONYME (26 novembre 1993): Anthony Burgess (obituary). The Times. 23.

4. Queneau, Raymond (1947): Exercices de style. Blanche. Paris: Gallimard.

5. Queneau, Raymond (1947/1983): Esercizi di stile. (Traduit du français par Umberto Eco) Turin: Einaudi.

6. «[...] non si trattava di tradurre almeno nel senso corrente del termine, ma di capire le regole di gioco che Queneau si era poste, e quindi giocare la stessa partita con un'altra lingua.» Quatrième de couverture de Queneau (1947/1983), traduit par Eco. La traduction française de cette citation est tirée de Henry, Jacqueline (2003): La traduction des jeux de mots. Paris: Presses Sorbonne Nouvelle, 108.

7. López Socasau, Federico et Ochoa Santamaría, Ignacio (1995): From Lost to the River. Madrid: Ediciones Formativas D.L.

8. Comme l'analyse implique plusieurs langues, et un Nadsat dans chacune d'entre elles, nous les distinguons comme suit: English, English Nadsat, français, Nadsat français, русский [russkij], русский Надсат [russkij nadsad], български [b"lgarski], български Надсат [b"lgarski nadsat]. Le gras fait ressortir les mots relevant du Nadsat.

9. Citation tirée d'une entrevue télévisée (date non spécifiée), reprise dans le documentaire The Age of Anxiety (1945-1969) (2010): Réalisé par Leith, Alexander. Épisode 2 de 3. In Their Own Words: British Novelists. BBC Four. Royaume-Uni.

10. Ce dernier chapitre est supprimé, contre le gré de Burgess, de la version américaine du livre, paru en 1963, sur laquelle Stanley Kubrick a basé son film. L'argument de l'éditeur était que les Américains, moins naïfs que les Anglais, ne croiraient pas à un «happy ending» de ce genre.

11. KAN, Alexandr (12 septembre 2012): «Заводной апельсин » : роману о садисте-эстете - 50 лет [«A Clockwork Orange»: le roman pour le sadique-esthète fête ses 50 ans]. ВBC News Рýсская слу́жба. Consulté le 6 avril 2017, <https://www.bbc.com/russian/multimedia/2012/09/120912_ clockwork_orange>.

12. Belmont et Chabrier traduisent gypsy par manouche.

13. Leur article et celui de Malamatidou (2017) s'inscrivent dans le cadre d'un projet intitulé «A Clockwork Orange Parallel Translation Corpus Project», qui a débuté en 2015 et qui vise, à travers 
l'analyse des traductions du Nadsat, à mettre en lumière certains défis auxquels les traducteurs de textes littéraires doivent faire face. Le blogue du projet offre une liste détaillée de tous les items du Nadsat relevés par cette analyse stylistique du corpus. Voir Clarke, Jim, Vincent, Benet, Malamatidou, Sofia et al. (Dernière mise à jour: 16 novembre 2017): Ponying the Slovos. A Clockwork Orange Parallel Translation Corpus Project. Consulté le 23 mai 2019, <https:// ponyingtheslovos.wordpress.com/>.

14. Rédacteur en chef de la revue Innostrannaâ literatura [Littérature étrangère], universitaire, professeur d'histoire de la littérature étrangère et de traduction littéraire.

15. Burgess, Anthony (18 septembre 1970): Bless Thee Bottom. Times Literary Supplement. 3577:102425.

16. «концептуальная нелепость, потому что русский жаргон служил автору для выражения идеи, что зло идет с Востока, из СССР, из России, которая считалась империей зла .

17. «иронически шифровал, задавал читателю простенькие ребусы, стараясь, чтобы слова звучали так, как будто их произносят иноязычные персонажи, которым русский язык совершенно чужд и в их устах он слегка режет ухо. Специально путал корни с суффиксами.»

18. Voir notamment la page Web consacrée à la traduction bulgare sur le site d'Helikon, où l'on trouve de nombreux commentaires d'utilisateurs. Consulté le 20 mai 2018, <https://m.helikon.bg/ books/76/-Портокал-с-часовников-механизъм_147250.html\#book_info>.

\section{RÉFÉRENCES}

Antoine, Fabrice (2004): Argots et langue familière: quelle représentation en lexicographie bilingue. In: Fabrice Antorne, dir. Argots, langue familière et accents en traduction. Villeneuve-d'Ascq: Université Charles-de-Gaulle-Lille 3/Maison de la recherche, 11-23.

Blonskyté, Marija et Petroniené, Saule (2013): Translation of the Russian Nadsat Slang in Anthony Burgess' Novel A Clockwork Orange into Lithuanian. Studies About Languages. $22: 62-70$.

Bogic, Anna (2017): Anthony Burgess in French Translation: Still "as Queer as a 'Clockwork Orange". In: Marc Jeannin, dir. Anthony Burgess and France. Cambridge: Cambridge Scholars Publishing, 215-227.

Breton, Dominique et Tinchant-Benrahro, Sabine (2014): Traduire le spanglish ou la 'fabrique du lecteur' en question. La main de Thôt. (2):14 p. Consulté le 20 février 2018, $<$ http://revues.univ-tlse2.fr/lamaindethot/index.php?id=270>.

Chan, Leo Tak-Hung (2002): Translating Bilinguality. The Translator. 8(1):49-72.

Clarke, Jim (2017): Lives in language: Anthony Burgess. Babel. 19:32-33.

Delesse, Catherine (2012): Présentation. In: Catherine Delesse, dir. Inscrire l'altérité: emprunts et néologismes en traduction. Palimpsestes. 25:9-19.

DerridA, Jacques (1982): Réponse à l'intervention de Patrick Mahony: «Transformations et déconstruction parricide». In: Claude LÉvesque et Christie V. MCDonald, dir. L'oreille de l'autre: otobiographies, transferts, traductions. Textes et débats avec Jacques Derrida. Montréal: VLB, 132-146.

Екimova-MelnišKa, Mariana (2009): Ключ за механизма на портокала (Klûč za mehanizma na portokala) [Clé pour le mécanisme de l'orange]. In: Anthony Burgess (1962/2009): Портокал с часовников механизбм (Portokal s časovnikov mehaniz"m) [Orange mécanique]. Sofia: Iztok-Zapad: 7-13.

Evans, Robert (1971): Nadsat: The Argot and its Implications in Anthony Burgess' 'A Clockwork Orange'. Journal of Modern Literature. 1(3):406-410.

Grutman, Rainier (1997): Des langues qui résonnent. L'Hétérolinguisme linguistique au $X I X^{e}$ siècle québécois. Montréal: Fides.

Grutman, Rainier (2012): Traduire l'hétérolinguisme: Questions conceptuelles et (con)textuelles. In: Marie-Annick Montout, dir. Autour d'Olivier Senior: hétérolinguisme et traduction. Angers: Presses de l'Université d'Angers, 49-81.

Haugen, Einar (1950): The Analysis of Linguistic Borrowing. Language. 20(2):210-231.

Henry, Jacqueline (2003): La traduction des jeux de mots. Paris: Presses Sorbonne Nouvelle. 
Humbley, John (2006): La néologie: interface entre ancien et nouveau. In: Rosalind GreensTEIN, dir. Langues et cultures: une histoire d'interface. Paris: Publications de la Sorbonne, 91-104.

JACKson, Howard (2011): Invented Vocabularies: The Cases of Newspeak and Nadsat. In: Michael Adams, dir. From Elvish to Klingon. Exploring Invented Languages, Oxford: Oxford University Press, 49-73.

Krumova-Cvetrova, Liliâ, Blagoeva, Diana, Kolkovska, Siâ et al. (2013): Българска лексикология [Lexicologie bulgare]. Vol. 1. Sofia: Presses académiques «Prof. Marin Drinov».

Lesiew, Kamil (2015): A Clockwork Orange: Ultra-violence Rewritten. Folio. 1(14):64-78.

Malamatidou, Sofia (2017): Creativity in translation through the lens of contact linguistics: a multilingual corpus of $A$ Clockwork Orange. The Translator. 23(3):292-309.

Martín Ruano, M. Rosario (2003): Bringing the other back home: the translation of (un) familiar hybridity. Linguistica Antverpiensia. 2:191-204.

MezeI, Kathy (1988): Speaking White: Literary Translation as a Vehicle of Assimilation in Quebec. Canadian Literature - Litterature canadienne. 117:11-24.

Montagut, Aina Lopèz (2014): Traduire une langue qui n'existe pas. La main de Thôt. 2:16 p. Consulté le 30 mars 2018, <http://revues.univ-tlse2.fr/lamaindethot/index.php?id=319>.

NidA, Eugene et TABer, Charles (1969/1982): The Theory and Practice of Translation. Linden: United Bible Societies.

Pochon, Jean (2010): Analyse de la traduction française de l'Orange mécanique: comment traduire la création lexicale? Mémoire de maîtrise non publié. Genève: Université de Genève. Consulté le 20 mai 2018, <http://archive-ouverte.unige.ch:14840>.

Rozental, Ditmar, Golub, Irina et Telenkova, Margarita (1994): Современный русский язык [Russe contemporain]. Moscou: Meždunarodnye otnošeniâ.

Sternberg, Meir (1981): Polylingualism as Reality and Translation as Mimesis. Poetics Today. 2(4):221-239.

Stratford, Madeleine (2008): Au tour de Babel! Les défis multiples du multilinguisme. Meta. 53(3):457-470.

Vincent, Benet et Clarke, Jim (2017): The language of A Clockwork Orange: A corpus stylistic approach to Nadsat. Language and Literature. 26(3):247-264.

Windle, Kevin (1995): Two Russian Translations of A Clockwork Orange, or the Homecoming of Nadsat. Canadian Slavonic Papers / Revue Canadienne des Slavistes. 37(1-2):163-185.

\section{ANNEXES}

\section{Annexe 1: CEuvres de Burgess}

Burgess, Anthony (1962): A Clockwork Orange. Portsmouth: Heinemann.

Burgess, Anthony (17 février 1972): Clockwork Marmalade. The Listener. 87(2238):197-199.

Burgess, Anthony (1962/1972) : L'orange mécanique. (Traduit de l'anglais par Georges Belmont et Hortense Chabrier) Le Livre de Poche. Paris: Éditions Robert Laffont.

Burgess, Anthony (1980): Earthly Powers. Londres: Hutchinson.

Burgess, Anthony (1980/1981): La Puissance des ténèbres. (Traduit de l'anglais par Georges Belmont et Hortense Chabrier) Paris: Acropole.

Burgess, Anthony (1990): You've Had Your Time. Portsmouth: Heinemann.

Burgess, Anthony (1962/1991): Заводной апельсин (Zavodnoĭ apel'sin) [Orange mécanique]. (Traduit de l'anglais par Evgenij Sine'ŝsıkov) Bishkek: Forum.

Burgess, Anthony (1962/1991): Заводной апельсин (Zavodnoŭ apel'sin) [Orange mécanique]. (Traduit de l’anglais par Vladimir BošNÂK) Leningrad: Hudožestvennaâ literatura.

Burgess, Anthony (1962/1991): Портокал с механизбм (Portokal s mehaniz"m) [Orange à mécanisme]. (Traduit de l'anglais par Mariana Eкıмova-MelnišKa) Varna: Galaktika.

Burgess, Anthony (1962/2000): A Clockwork Orange. Londres: Penguin Books. 
Burgess, Anthony (1962/2009): Портокал с часовников механизбм (Portokal s časovnikov mehaniz"m) [Orange mécanique]. (Traduit de l’anglais par Mariana MeLnišKa) 2e éd. Sofiâ: Iztok-Zapad.

\section{Annexe 2: Les traducteurs dans la presse}

BošNÂк, Vladimir (2010): “Собачая цепь на дубе том” Интервю с Владимиром Бошняком (Sobačaâ cep' na dube tom) [ «L chaîne du chien fixée à ce chêne». Entretien avec Vladimir Bošnâk]. (Entretien par Elena KalAšniкova) Иностранная литература (Inostrannaya literatura) [Littérature étrangère]. 1. Consulté le 26 mars 2016, <http://magazines.russ.ru/ inostran/2010/1/so7.html>.

BošNÂк, Vladimir (18 novembre 2015) : Владимир Бошняк: «Настоящей литратуры просто больше нет» (Vladimir Bošnâk: «Nastoâsej litratury prosto bol'še net») [Vladimir Bošnâk: «La vraie littérature n'existe tout simplement plus». (Entretien par Daniil Adamov) m24. ru. Consultéle 31 janvier 2017, <http://www.m24.ru/articles/90130 ?utm_source=CopyBuf >).

Eкıмova-MelnišKa, Mariana (8 février 2010) : Мариана Мелнишка: «Най-прекрасното днес е, че имаме избор» (Mariana Melniška: «Naj-prekrasnoto dnes e, če imame izbor») [Mariana Melniška: «Le plus formidable aujourd'hui, c'est que nous avons le choix»]. (Entretien par Maria Duкovsкa) Az-jenata.bg. Consulté le 20 juin 2017, <http://www.azjenata.bg/a/15-tya-zvezdata/9661-mariana-melnishka-nay-prekrasnoto-dnes-e-che-imameizbor/>. 